\title{
Optimal lane expansion model for electric vehicle transportation networks with bounded rational travelers
}

\section{Xu Xin}

School of Economics and Management, Tongji University https://orcid.org/0000-0002-0780-156X

\section{Xiaoli Wang}

School of Economics and Management, Tongji University

\section{Kang Chen}

School of Maritime Economics and Management, Dalian Maritime University

Cui Li

School of Mechanical Engineering, Tongji University

Yanran Liu ( $\nabla$ yanranliu@tongji.edu.cn )

School of Economics and Management, Tongji University

\section{Research Article}

Keywords: Network design problem, Battery electric vehicle (BEV), Range anxiety, Bounded rationality, Active set algorithm (ASA)

Posted Date: February 10th, 2022

DOI: https://doi.org/10.21203/rs.3.rs-1339708/v1

License: (c) (i) This work is licensed under a Creative Commons Attribution 4.0 International License. Read Full License 


\section{Optimal lane expansion model for electric vehicle transportation networks with bounded rational travelers}

Xu Xin ${ }^{\mathrm{a}}$, Xiaoli Wang ${ }^{\mathrm{a}}$, Kang Chen ${ }^{\mathrm{b}}, \mathrm{Cui} \mathrm{Li}^{\mathrm{c}}$ and Yanran $\mathrm{Liu}^{\mathrm{a}}$, d, *

${ }^{a}$ School of Economics and Management, Tongji University, Shanghai, 200092, PR

China, ${ }^{b}$ School of Maritime Economics and Management, Dalian Maritime University,

Dalian, Liaoning, 116026, PR China; 'School of Mechanical Engineering, Tongji

University, Shanghai, 201804, PR China; ${ }^{d}$ Department of Logistics and Maritime

Studies, The Hong Kong Polytechnic University, Hong Kong

E-mail: xinxu@tongji.edu.cn (X. Xin), xiaoli-wang @tongji.edu.cn (X. Wang),

chenkang@dlmu.edu.cn (K. Chen), licui20140718@tongji.edu.cn (C. Li),

yanranliu@tongji.edu.cn (Y. Liu).

ORCID: 0000-0002-0780-156X (X. Xin), 0000-0003-1484-1209 (K. Chen), 00000002-6952-1416 (Y. Liu).

E-mail for the *corresponding author: yanranliu @tongji.edu.cn 


\title{
Optimal lane expansion model for electric vehicle transportation networks with bounded rational travelers
}

\author{
With governments worldwide emphasizing environmental protection and the \\ global focus on carbon reduction, the battery electric vehicle (BEV) industry has \\ developed rapidly. Urban transportation network with BEVs as the main form of \\ transportation will soon be formed. Thus, we investigate a BEV transportation \\ network design problem, and a lane expansion model is established. The \\ abovementioned model aims to minimize the system travel time of BEV \\ transportation networks and optimize government lane expansion schemes under \\ limited budgets. To consider the travel characteristics of BEV drivers, range \\ anxiety and bounded rationality factors are simultaneously incorporated into the \\ model framework. A heuristic algorithm is designed based on the active set \\ algorithm to obtain the local optimal solution of the actual scale problem. \\ Moreover, a column generation technique is embedded in the abovementioned \\ algorithm to avoid the enumeration of paths. Sensitivity analyses of different \\ levels of rationality and government investment scales are performed. The \\ experimental results demonstrate that the abovementioned model and algorithm \\ effectively solve the considered problem and provide decision support for the \\ government in formulating transportation planning policies.
}

JEL Code: C61; D58; H54; R42; R53

Keywords: Network design problem; Battery electric vehicle (BEV); Range anxiety; Bounded rationality; Active set algorithm (ASA)

\section{Introduction}

As reported by the World Meteorological Organization (WMO), in the past 50 years, more than 11,000 disasters caused by weather, climate, and water have caused 2 million deaths and 3.6 trillion US dollars in economic losses, and nearly 22 million people have become climate refugees (World Meteorological 2021). Carbon emissions are becoming a major practical issue affecting human survival and development (Chu et al. 2021; Zhao et al. 2020). Social and economic development processes have simultaneously put 
considerable pressure on the natural environment. It should be noted that global carbon dioxide emissions will continue to grow with the future growth of the economy, population and resource demand. Therefore, mitigating greenhouse gas emissions is among the most urgent and far-reaching issues in the international political economy, and the sustainable development of high-energy-consumption and high-carbon-emission industries, such as the transportation industry, must be promoted.

In recent years, with the strict management and control of carbon emissions by the governments of various countries, the battery electric vehicle (BEV) manufacturing industry has attracted considerable research attention (Xu et al. 2021). Substituting BEVs for traditional gasoline vehicles $(\mathrm{GVs})$ is an effective way to reduce greenhouse gas $(\mathrm{GHG})$ emissions and the dependence of the automobile industry on petroleum $(\mathrm{Hu}$ et al. 2019; Waraich et al. 2013). Therefore, various countries have announced plans to achieve carbon neutrality by the middle of the $21^{\text {st }}$ century, and their governments have introduced policies to promote the BEV industry; consequently, the production of BEVs has increased rapidly. According to data released by Statistics Canada, the number of newly registered BEVs nationwide increased from 25,163 in 2014 to 69,010 in 2018, a growth rate of $174 \%$ (Lin et al. 2021). In China, the number of BEV and plug-in hybrid vehicle (HEV) registrations increased from 1,430 in 2010 to 579,000 in 2017 (Cheng et al. 2020). Thus, BEVs will be a major part of future urban transportation networks. Not surprisingly, BEV transportation network design has become the focus of both the government and academia because it promotes green transportation (Montoya et al. 2016; Schiffer and Walther 2017).

In a BEV transportation network, the behavior of travelers is often different from that in a traditional GV transportation network. On the one hand, range anxiety makes the feasible travel path of BEV drivers significantly different from that of GV 
drivers. Range anxiety reflects travelers' fear of running out of battery energy before reaching their destination (or charging station) (Xu et al. 2020; Yuan et al. 2018). Due to the range anxiety phenomenon, travelers are concerned about their battery state-ofcharge (SOC), and they will make prudent charging decisions (Cheng et al. 2020; Miwa et al. 2017). It is manifested in the fact that travelers often do not allow the SOC of BEVs to be lower than a certain level during travel (Neubauer and Wood 2014). Under the influence of range anxiety, BEV drivers will choose the most secure travel route that ensures an available power supply (Xu et al. 2017; Yang et al. 2016). This constraint allows us to establish a different definition of feasible paths in the design of BEV transportation networks.

On the other hand, traveler behaviors are often associated with bounded rationality. In the traditional transportation network design problem, it is assumed that travelers choose transportation routes based on decision rules for utility maximization; i.e., the traffic flow distribution in the transportation network obeys the perfect rational user equilibrium (PRUE) principle. However, this assumption has been challenged by numerous scholars (Ilin and Rogova 2017; KAI-INEMAN and Tversky 1979). Researchers have found that when choosing a departure time (Mahmassani and Chang 1986; Schwanen and Ettema 2009) or a transportation route (Zhou et al. 2014), travelers' choices are often associated with bounded rationality. Under the influence of the above phenomenon, the traffic flow distribution in a transportation network then forms a bounded rational user equilibrium (BRUE) state: an equilibrium state in which travelers' adjustments to travel routes will not cause their travel time to exceed an established limit (Eikenbroek et al. 2018; Mahmassani and Chang 1987). From an economic perspective, bounded rationality is associated with people's habits, inertia (Lotan 1997; Samuelson and Zeckhauser 1988), and myopia (Bogers et al. 2005). With 
bounded rationality, the equilibrium state of a BEV transportation network can highly vary from that of a traditional GV transportation network.

Motivated by the two abovementioned behavioral factors, in-depth research on the design of BEV transportation networks is conducted in this paper. Our paper aims to investigate a $\boldsymbol{B} E V$ transportation Network Design Problem considering two human choice behaviors (BNDP) to help governments better develop lane expansion schemes for specific regions. Specifically, the equilibrium conditions of the transportation network are first developed to describe the BRUE state of the BEV network. Then, a network design model is constructed to optimize the government's optimal lane expansion scheme and to minimize the system travel time. Finally, to solve the abovementioned model, a heuristic algorithm is designed to identify the local optimal solution based on the framework of the active set algorithm (ASA). Moreover, a column generation algorithm (CGA) is embedded in the abovementioned algorithm to avoid the enumeration of paths.

The contributions of this paper are threefold. First, we define a new BEV transportation network design problem. To the best of our knowledge, our paper is the first to design a BEV transportation network considering both range anxiety and BRUE factors. Second, we establish the relevant equilibrium conditions for the BRUE-based transportation network and extend the theory of bounded rationality in economics to the field of BEV transportation network design. Third, we design a heuristic algorithm that explores the local optimal solution of the problem based on an ASA framework. The abovementioned algorithm can provide an efficient solution for problems at practical scales.

The remainder of this paper is organized as follows. Section 2 reviews the literature related to BEV transportation network equilibrium and network design to 
clarify the current research gaps. Section 3 presents the assumptions, problem descriptions, and mathematical expressions associated with the BRUE state of the BEV transportation network. Section 4 models the BEV transportation network design problem considering range anxiety and BRUE factors. The algorithm for solving the model is presented in Section 5. In Section 6, numerical experiments are performed to verify the effectiveness of the model and algorithm. Finally, Section 7 concludes the paper and proposes several potential research directions.

\section{Literature review}

Research on the design of BEV transportation networks is inseparable from the discussion of the equilibrium state of the networks. This is because the calculation of the equilibrium state of a transportation network is the basis for evaluating the design scheme of the network. The concept of transportation network equilibrium was first defined by Wardrop in 1952 (Wardrop 1952). Wardrop (1952) assumed that all drivers choose their travel route based on the principle of the shortest travel time and proposed the famous Wardrop's first principle. In recent years, with the growth of BEV ownership, research on the equilibrium state of BEV transportation networks has expanded. In the early stage, He et al. (2014) first proposed a BEV transportation network equilibrium model considering range anxiety. The authors noted that the difference between the equilibrium state of a BEV transportation network and that of a GV network is associated with the phenomenon of range anxiety, and range anxiety seems unrealistic to eliminate. Based on the consideration of the above phenomenon, the authors constructed two network equilibrium models, one of which further considered flow-dependent energy consumption.

Considering the characteristics of wireless charging lanes, Chen et al. (2016) established a comparatively complicated BEV transportation network equilibrium 
model. The authors assumed that BEV drivers could choose to charge wirelessly in a charging lane. To ensure that a BEV has sufficient battery power, the driver can adjust the charge received by changing the travel speed of the vehicle. Xu et al. (2017) suggested that the conditions proposed by He et al. (2014) were incomplete, and they constructed a set of network equilibrium conditions. These conditions allowed both BEVs and GVs to exist in the studied transportation network. To reflect real-world conditions in the model, the authors further introduced the road grade effect. When a BEV is driving on roads of different grades, the dwell time and swapping cost will vary. However, the authors assumed that only swapping stations existed in the studied transportation network. This assumption limited the impact of dwell time on network equilibrium during the modeling process. Liu and Song (2018) developed a BEV transportation network equilibrium model considering flow-dependent electricity consumption. Unlike Xu et al. (2017), the authors proposed a solvable mathematical model rather than several equilibrium conditions. In this way, the equilibrium state of the transportation network could be obtained by solving the model directly. Recently, Chen et al. (2020) introduced queuing theory in conjunction with a set of network equilibrium conditions and constructed a BEV transportation network equilibrium model considering the BEV queuing time at charging stations. To solve the proposed mathematical program with complementarity constraints (MPCC), the authors converted the original model into a sequence of relaxed nonlinear programming problems. Generally, when scholars have constructed sets of equilibrium conditions for BEV transportation networks, they have tried to accurately model the relationships among vehicle flows, energy consumption and queuing behaviors at transportation nodes. However, in characterizing the route choice behaviors of travelers, scholars often simply assume that each traveler is perfectly rational. In practice, bounded rationality is 
an important characteristic related to travelers in a transportation network, and BEV transportation networks are not exceptions.

According to different definitions of the equilibrium state of BEV transportation networks, scholars have conducted extensive research on transportation network design problems. A typical representative transportation network design problems involves the layout of charging facilities (or other infrastructure). For example, Qiu et al. (2020) addressed an optimization problem for the layout design of an electrified road for electric vehicles. Based on the classic UE theory proposed by Wardrop (1952), the authors established the equilibrium conditions of the transportation network and constructed a network design model. To solve the problem at the actual network scale, the authors designed a modified ASA. Cheng et al. (2020) considered the charging time of BEVs and range anxiety factors and constructed a network design model based on the equilibrium conditions proposed by He et al. (2014). The authors aimed to minimize the system travel time and to optimize the government's lane expansion scheme to limit traffic congestion. Furthermore, a robust optimization model was constructed considering the uncertainty of the transportation demand. Chen et al. (2016) assumed that BEV drivers can adjust their driving speeds on electrified roads to increase the charge they received. Based on the equilibrium conditions proposed, the authors discussed the government's optimal link reconstruction scheme (in which links are converted to electrified roads) under a limited budget. Liu and Song (2018) further considered the abovementioned factors and constructed a congestion toll model. The authors verified the nonuniqueness of the vehicle flow distribution, and robust optimization theory was introduced into the research framework to improve the worstcase scenario for the traffic flow distribution. Unfortunately, the authors assumed that 
travelers' choices of transportation routes obey the PRUE principle; this is equivalent to ignoring the impact of the irrational behavior of travelers on travel time in the network.

Based on the abovementioned studies, two research gaps are identified. First, the existing research has not broadly focused on network equilibrium or BEV transportation network design considering the bounded rationality of travelers; thus, studies of BRUE in BEV transportation networks have been inadequate. Second, algorithm development for network design models with bounded rationality has been limited, especially for BEV transportation networks. Notably, algorithms that can obtain local (or global) optimal solutions to problems have not been studied in detail. Therefore, to fill these research gaps, we first define the bounded rationality of travelers in BEV transportation networks and then give a mathematical expression of the BRUE state in these networks. Next, a mathematical model is constructed to solve the transportation network design problem. Finally, considering the characteristics of the model, a heuristic algorithm is designed to obtain a solution. Overall, the above model and algorithm can provide necessary decision support for the government to rationally construct transportation infrastructure.

\section{Problem description}

\subsection{Assumptions and notations}

To facilitate modeling, we introduce the basic assumptions (or considerations) for the BNDP.

(1) Behavior of BEV drivers: When travelers choose transportation routes, they will follow the principle of bounded rationality. Under the influence of the abovementioned principle, when the difference between the travel times along two paths is within a threshold value, these paths are considered alternative 
paths for travelers. The network equilibrium state based on this travel choice behavior is called the BRUE state.

(2) Charging time of BEVs: Unlike traditional GVs, BEVs require charging times that generally take several hours. Therefore, if a BEV needs to be charged during travel, the charging time needs to be added to the travel time. We assume that the BEV driver can charge their BEV at a charging station in the transportation network used for travel and that the charging time is proportional to the amount of battery that must be charged. In a BEV transportation network, each charging station has only one type of charging pile, and the number of charging piles at a charging station is assumed to be sufficient. In other words, we do not consider the case of BEV queuing at charging stations.

(3) BEV energy consumption and the range anxiety of travelers: Each BEV has a fixed battery storage capacity; thus, we assume that each BEV has the same initial state of charge (i.e., the SOC of the battery before the trip). Note that this assumption can be relaxed by introducing different types of BEVs. The battery power will decrease linearly as the transportation distance increases. Due to the existence of range anxiety, BEV drivers will not allow the SOC of the battery to fall below a predetermined value at any time.

(4) Link performance function: The function between the traffic flow and the transportation time along each link is called the performance function. We assume that in the BEV transportation network, the performance function for each link follows the form of the Bureau of Public Roads (BPR) function. Moreover, the travel times on different links are additive.

(5) The government's decision: The government aims to establish a reasonable lane expansion scheme with the goal of minimizing the system travel time under a 
limited budget. We assume that the government will not construct additional links and will only expand lanes based on existing links. In addition, for modeling convenience, we assume that the government expands each link by 3 lanes at most. The above assumptions can also be relaxed by introducing additional binary variables.

For convenience, the notations frequently used in this paper are given as follows.

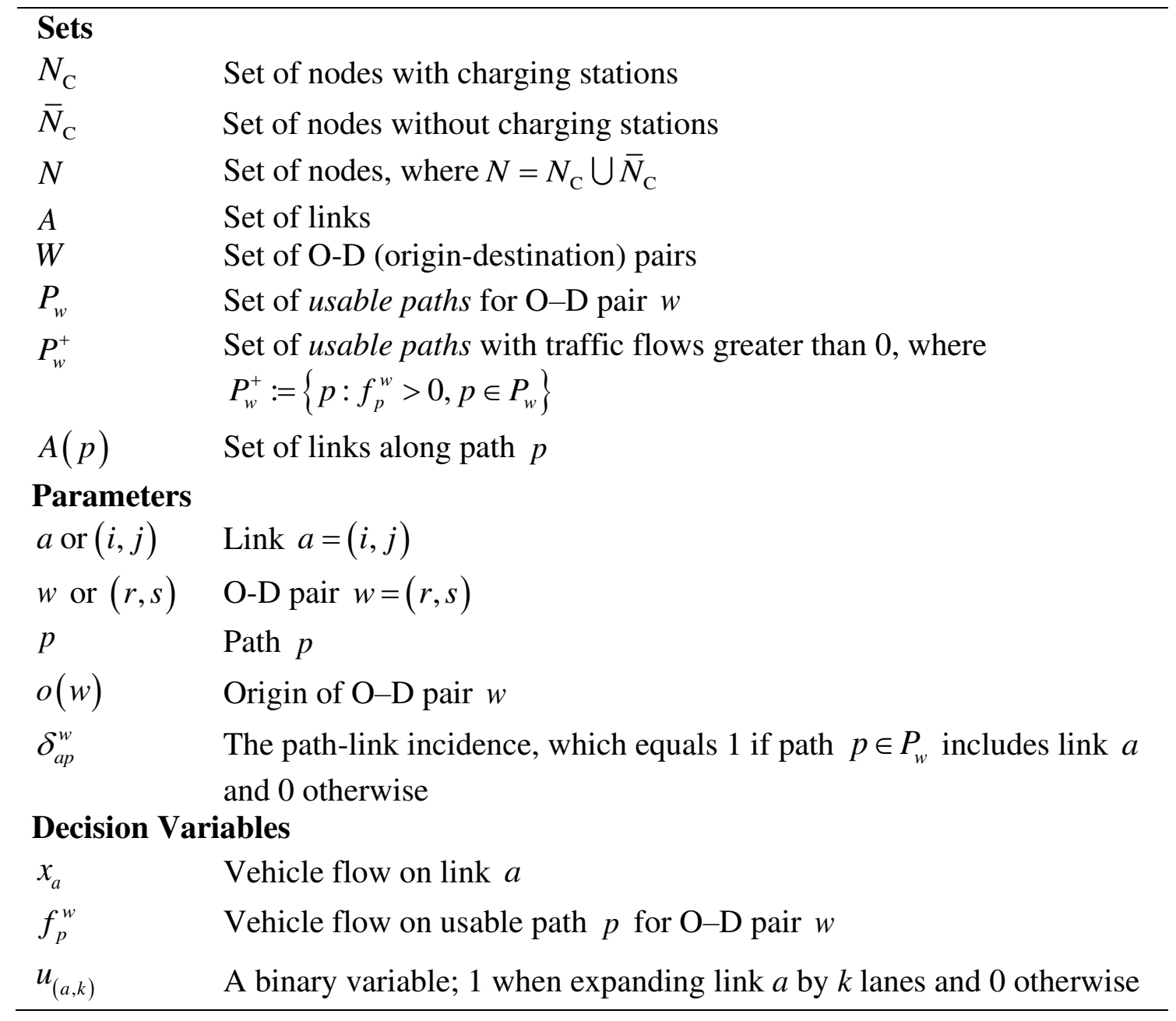

\subsection{BEV transportation network formulation}

Let $G:=(N, A)$ denote a BEV transportation network. $G$ is composed of a node set $N$ and a link set $A$. Different from a traditional transportation network, in this BEV 
transportation network, there are charging stations at the nodes. To describe the above situation, we let $N:=N_{\mathrm{C}} \cup \bar{N}_{\mathrm{C}}$, where $N_{\mathrm{C}}$ represents the set of nodes with charging stations and $\bar{N}_{\mathrm{C}}$ denotes the set of nodes without charging stations. Moreover, we introduce Equation (1) to describe the travel time of BEVs along each link. This equation has the same form as the BPR function. In the equation, we let $a:=(i, j)$ represent a link, where $j$ and $i$ are the head node and tail node of link $a$, respectively. $v_{a}$ denotes the flow volume on link $a$, and $\mathbf{u}=\left(u_{(a, k)}\right)$ is the lane expansion scheme for the BEV transportation network designed by the government, where $u_{(a, k)}$ is a binary variable. When $u_{(a, k)}$ is equal to 1 , the government expands link $a$ by $k$ lanes. It should be noted that we only consider two cases, $k=1$ and $k=2$, to match our assumption that each link can only expand to 3 lanes. In other cases with higher $k$ values, the developed model could be applied to expand routes to any number of lanes. $t_{a}^{\text {free }}$ denotes the freeflow travel time on link $a . \alpha_{1}$ and $\alpha_{2}$ are two positive coefficients to be predetermined. $C A_{a}$ and $C A_{a}^{\text {unit }}$ represent the current transportation capacity of link $a$ and the increase in capacity after expanding the link by one lane, respectively.

$$
t_{a}\left(v_{a}, u_{(a, k)}\right)=t_{a}^{\text {free }}\left[1+\alpha_{1}\left(\frac{v_{a}}{C A_{a}+C A_{a}^{\text {unit }}\left(u_{(a, 1)}+2 u_{(a, 2)}\right)}\right)^{\alpha_{2}}\right]
$$

\section{Model establishment}

\subsection{Formulation of the BRUE state}

Now, we discuss the BRUE state in the BEV transportation network. In this paper, we assume that all vehicles in the transportation network are BEVs. Notably, with the strict 
environmental policies being established by various governments, BEVs will come to dominate transportation networks in the foreseeable future. According to the principle of bounded rationality, each traveler can choose their travel path freely when the travel times along several paths do not exceed a predetermined threshold. However, due to battery power restrictions and range anxiety, some paths will be inappropriate. To describe the above situation, we introduce the following definitions.

Definition 1 (Useable path). A path is useable if and only if the traveler can complete the journey by selecting this path and the SOC of the battery will not cause the traveler to feel range anxiety at any time during the trip (He et al. 2014).

Definition 1 indicates that in a BEV transportation network, the SOC at any point along a usable path must be greater than or equal to the minimum battery power that causes range anxiety. Figure 1 further illustrates Definition 1.

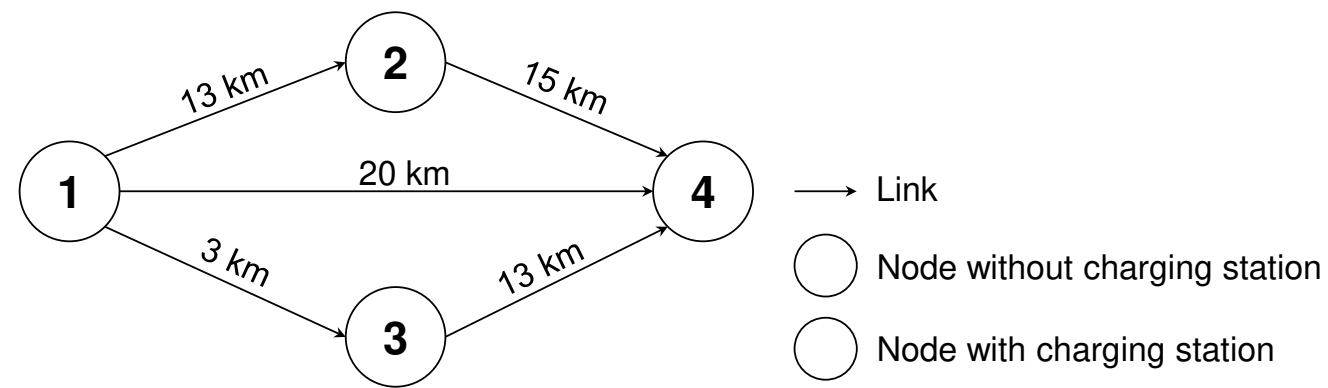

Fig. 1. Schematic diagram of a BEV transportation network

A BEV network with 4 nodes and 5 links is shown in Figure 1, and the length of each link is marked above it. There are charging stations at Node 2 and Node 3 . It is assumed that the traveler's trip goes from Node 1 to Node 4 . Therefore, there are three path options: Path 1: 1-2-4, Path 2: 1-4, and Path 3: 1-3-4. We set the initial SOC of the traveler's BEV to $15 \mathrm{kWh}$ and the electricity consumption of the BEV to $1 \mathrm{kWh}$ per kilometer. Without considering range anxiety, Path B becomes unusable because the battery power consumed by the BEV on link $(1,4)$ is $20 \mathrm{kWh}$. When we define the range anxiety of travelers as $1 \mathrm{kWh}$, Path A further becomes unusable. This is because 
after the driver travels 14 kilometers on link $(2,4)$, the battery power will be less than 1 $\mathrm{kWh}$. Similarly, if their range anxiety is further increased to $3 \mathrm{kWh}$, the traveler will not be able to reach Node 4 from Node 1 because all paths are unusable. Based on Definition 1, we give the following two definitions to describe the BRUE state of the BEV transportation network.

Definition 2 (Feasible path). For each origin-destination (O-D) pair, a usable path defined in Definition 1 is feasible if and only if the difference between the travel time along this path and the travel time along the fastest path is no larger than a threshold value.

Definition 3 (Network BRUE state). The traffic flow distribution in the BEV transportation network reaches the BRUE state if and only if all transportation demands are satisfied and each vehicle travels along a feasible path, as defined in Definition 2.

According to the abovementioned definitions, travelers may not choose the path with the shortest travel time when traveling. To model the BRUE state, we let $w$ denote an O-D pair and $W$ represent the set consisting of all O-D pairs. $p$ represents a useable path defined in Definition 1. $P_{w}$ denotes the set of all usable paths for O-D pair $w$. For every path $p \in P_{w}$, we further let $f_{p}^{w}$ be the traffic volume. $c_{p}^{w}$ represents the shortest charging time for travelers on useable path $p$ without range anxiety occurring; $\delta_{a p}^{w}$ denotes a path-link incidence, which equals 1 if path $p \in P_{w}$ consists of link $a \in A$ and 0 otherwise. $q_{w}$ represents the transportation demand between O-D pair $w$. Finally, we assume BEV drivers with the same transportation demand (i.e., same O-D pair) have the same threshold value of bounded rationality, denoted as $\bar{\varepsilon}^{w}$. Thus, the following proposition can be established. 
Proposition 1. Vector $\mathbf{f}=\left(f_{p}^{w}\right)$ denotes a BRUE traffic flow distribution if and only if for each O-D pair $w$, a $\lambda^{w}$ solution exists that satisfies the following equilibrium conditions:

$(1)$,

$$
\begin{array}{ll}
\bar{c}_{p}^{w} \geq \lambda^{w} & \forall p \in P_{w} \\
\bar{c}_{p}^{w} \leq \lambda^{w}+\bar{\varepsilon}^{w} & \forall p \in P_{w}^{+}:=\left\{p: f_{p}^{w}>0, p \in P_{w}\right\} \\
\sum_{p \in P_{w}} f_{p}^{w}=q_{w} & \\
v_{a}=\sum_{w \in W} \sum_{p \in P_{w}} f_{p}^{w} \delta_{a p}^{w} & \forall a \in A \\
\bar{c}_{p}^{w}=\sum_{a \in A(p)} t_{a}\left(v_{a}, u_{(a, k)}\right)+c_{p}^{w} & \forall p \in P_{w}
\end{array}
$$

where $\mathbf{f}=\left(f_{p}^{w}\right) \geq 0$.

Proof. See the proof in Section 2.1 of Lou et al. (2010) for details (Q.E.D.).

However, when applying the abovementioned network equilibrium conditions, all paths need to be enumerated due to the existence of Constraint (3). To solve the model, we further introduce a slack variable $\varepsilon_{p}^{w}$. Then, the abovementioned BRUE conditions (1) to (6) can be transformed as follows.

(1), (5),

$$
\begin{array}{ll}
\bar{c}_{p}^{w}-\varepsilon_{p}^{w}-\lambda^{w}=0 & \forall p \in P_{w}, w \in W \\
f_{p}^{w}\left(\bar{\varepsilon}^{w}-\varepsilon_{p}^{w}\right) \geq 0 & \forall p \in P_{w}, w \in W \\
\sum_{p \in P_{w}} f_{p}^{w}=q_{w} & \forall w \in W \\
\bar{c}_{p}^{w}=\sum_{a \in A(p)} t_{a}\left(v_{a}, u_{(a, k)}\right)+c_{p}^{w} & \forall p \in P_{w}, w \in W
\end{array}
$$


where $\boldsymbol{\varepsilon}=\left(\varepsilon_{p}^{w}\right) ;(\mathbf{f}, \boldsymbol{\varepsilon}) \geq 0$.

Remark. We can easily verify that when $\bar{\varepsilon}^{w}$ equals 0 , the variable $\lambda^{w}$ represents the equilibrium travel time for O-D pair $w$ in the PRUE state. When $\bar{\varepsilon}^{w}>0$, the traffic flow distribution that satisfies (1), (5), and (7)-(10) is a BRUE distribution according to Definition 3. $\lambda^{w}$ represents the shortest travel time for O-D pair $w$, and $\varepsilon_{p}^{w}$ denotes the difference between the travel time along path $p$ and the shortest time $\lambda^{w}$.

\subsection{Finding best- and worst-case scenarios for a network}

In the BRUE state, the traffic flow along each link is not unique because travelers can choose arbitrarily from several feasible paths. This uncertainty poses a challenge for the government in transportation network design. However, among the numerous choices of travelers, there are two extreme situations: the shortest system travel time case (best case) and the longest system travel time case (worst case). This section focuses on the abovementioned two extreme cases in BEV transportation networks because the traffic flow distributions in these two situations can provide estimates of the effectiveness of the BEV transportation network design. Referring to the modeling method proposed by Liu and Song (2018), we construct a network equilibrium model as an MPCC to facilitate the design of the corresponding solution algorithm. To transform Constraint (8) into an equilibrium constraint, we introduce a slack variable $y_{p}^{w}$. Thus, the model that can solve the best/worst-case problems for the BRUE state of the BEV transportation network is established as follows. Note that Constraint (8) is converted into Constraints (12) to (14) in this case.

BC/WC-BRUE:

$$
\min _{(\mathbf{v}, \mathbf{f}, \mathbf{y}, \mathbf{\varepsilon}, \lambda)} \sum_{a \in A} \sum_{k=1,2} t_{a}\left(v_{a}, u_{(a, k)}\right) v_{a}+\sum_{w \in W} \sum_{p \in P_{w}} c_{p}^{w} f_{p}^{w}
$$


s.t. (1), (5), (7), (9), and (10),

$$
\begin{array}{ll}
f_{p}^{w} y_{p}^{w}=0 & \forall w \in W, p \in P_{w} \\
y_{p}^{w} \geq \varepsilon_{p}^{w}-\bar{\varepsilon}^{w} & \forall w \in W, p \in P_{w} \\
y_{p}^{w} \geq 0 & \forall w \in W, p \in P_{w}
\end{array}
$$

where $(\mathbf{f}, \boldsymbol{\varepsilon}, \mathbf{o}) \geq 0 ; \mathbf{v}=\left(v_{a}\right) ; \mathbf{y}=\left(y_{p}^{w}\right)$ and $\lambda=\left(\lambda^{w}\right)$.

\subsection{Robust BEV network design model}

For real-world decision-making problems, if a decision-making scheme can improve upon the worst-case result, then we consider the scheme robust. Since the government is often cautious when formulating policies, we provide a robust optimization solution as a powerful decision-making reference for the government. A robust optimization model for solving the BNDP (BNDPM) is given in this section. Before introducing the model, we define the following notations. Let $\eta_{a}$ represent the investment required to expand link $a$ by one lane and $B$ denote the upper limit of government investment. Then, the BNDPM can be expressed as follows.

BNDPM:

$$
\begin{aligned}
& \min _{\mathbf{u}} \max _{(\mathbf{v}, \mathbf{f}, \mathbf{y}, \boldsymbol{\varepsilon}, \lambda)} \sum_{a \in A} \sum_{k=1,2} t_{a}\left(v_{a}, u_{(a, k)}\right) v_{a}+\sum_{w \in W} \sum_{p \in P_{w}} c_{p}^{w} f_{p}^{w} \\
& \text { s.t. (1), (5), (7), (8), (9), (10), (12), (13), and (14), } \\
& \sum_{a \in A} \eta_{a}\left(u_{(a, 1)}+2 u_{(a, 2)}\right) \leq B \\
& 0 \leq u_{(a, k)} \leq 1 \quad \forall a \in A, k=1,2 \\
& u_{(a, k)}\left(1-u_{(a, k)}\right)=0 \quad \forall a \in A, k=1,2
\end{aligned}
$$

where $\mathbf{u}=\left(u_{(a, k)}\right)$ 
The BNDPM is established as a min-max programming model. Equation (15) is the objective function, and the lane expansion scheme $\mathbf{u}$ must minimize the worst-case scenario (i.e., the flow distribution associated with longest system travel time) in the BEV transportation network. Constraint (16) restricts the government's investment from exceeding the budget ceiling. Constraints (17) and (18) ensure that the variable $u_{(a, k)}$ is binary.

To facilitate the description, we divide the objective function of the BNDPM into two parts: the inner problem and the outer problem. The inner problem refers to the maximized part of the objective function (15); i.e., $\max _{(\mathbf{v}, \mathbf{f}, \mathbf{y}, \mathbf{\varepsilon}, \lambda)} \sum_{a \in A} \sum_{k=1,2} t_{a}\left(v_{a}, u_{(a, k)}\right) v_{a}+$ $\sum_{w \in W} \sum_{p \in P_{w}} c_{p}^{w} f_{p}^{w}$. Moreover, we let $\Gamma(\mathbf{u}), \Theta$, and $\psi(\Theta)$ represent the feasible region, decision variable vector, and objective function of the inner problem of the BNPDM, respectively. Then, the inner problem of the BNPDM can be equivalently expressed in the following concise form, denoted as BNDPM-IN.

$$
\text { BNDPM-IN: } \Omega(\mathbf{u})=\underset{\Theta}{\arg \max }\{\psi(\Theta): \Theta \in \Gamma(\mathbf{u})\}
$$

Based on the mathematical expression proposed above, we can convert the original BNDPM into BNDPM-R, as shown below.

\section{BNDPM-R:}

$$
\min _{\mathbf{u}} \Omega(\mathbf{u})
$$

Subject to $\Omega(\mathbf{u})=\underset{\Theta}{\arg \max }\{\psi(\Theta): \Theta \in \Gamma(\mathbf{u})\}$ and Constraints (16) to (18).

Through analyzing BNDPM-R, we can observe a certain connection between the inner problem and the outer problem. The feasible region of the inner problem $\Gamma(\mathbf{u})$ is determined by the decision variable (vector $\mathbf{u}$ ) of the outer problem. The 
abovementioned relationship makes the BNDPM (or BNDPM-R) a generalized semiinfinite min-max problem (Polak and Royset 2005). Solving such problems has always been a challenge. To date, only a few studies (e.g., Royset et al. (2003) and Polak and Royset (2005)) have discussed the solution to this kind of problem. Moreover, the inner problem is an MPCC because of the existence of Constraints (12) to (14). This characteristic further violates the Mangasarian-Fromovitz constraint qualification at any feasible point in the set $\Gamma(\mathbf{u})$. Therefore, the algorithms proposed by previous scholars may not be suitable for solving the BNDPM or BNDPM-R. To obtain an effective solution to the above model, we refer to the method proposed by Lou et al. (2010) and design a heuristic method, as described in Section 5.

\section{Solution approach}

\subsection{Solving the best- and worst-case}

In this section, we solve for the BRUE state of the BEV transportation network based on the modified ASA proposed by Lou et al. (2010). The core concept of the ASA is to rewrite the two parts of one equilibrium condition into two different constraints. For example, in BC/WC-BRUE, the mathematical expression of the equilibrium condition (12) can be rewritten as shown in Equation (19).

$$
0 \leq f_{p}^{w} \perp y_{p}^{w} \geq 0 \quad \forall w \in W, a \in A
$$

Notably, the equilibrium condition consists of two parts: $f_{p}^{w}$ and $y_{p}^{w}$. Therefore, we develop two active sets, namely, $\Phi_{\mathrm{P}}:=\left\{(w, p): f_{p}^{w}=0, \forall p \in P_{w}, w \in W\right\}$ and $\Phi_{\overline{\mathrm{P}}}:=\left\{(w, p): y_{p}^{w}=0, \forall p \in P_{w}, w \in W\right\}$, according to these two parts. Based on $\Phi_{\mathrm{P}}$ and $\Phi_{\overline{\mathrm{P}}}, \mathrm{BC} / \mathrm{WC}-\mathrm{BRUE}$ can be reformulated as the following model, denoted as RBC/WC-BRUE. 


\section{R-BC/WC-BRUE:}

$$
\min _{(\mathbf{v}, \mathbf{f}, \mathbf{y}, \mathbf{\varepsilon}, \lambda)} \sum_{a \in A} \sum_{k=1,2} t_{a}\left(v_{a}, u_{(a, k)}\right) v_{a}+\sum_{w \in W} \sum_{p \in P_{w}} c_{p}^{w} f_{p}^{w}
$$

s.t. (1), (5), (7), (9), (10), (13), and (14),

$$
\begin{array}{ll}
f_{p}^{w}=0 & \forall(w, p) \in \Phi_{\mathrm{P}}^{\sigma} \\
y_{p}^{w} \geq 0 & \forall(w, p) \in \Phi_{\mathrm{P}}^{\sigma} \\
f_{p}^{w} \geq 0 & \forall(w, p) \in \Phi_{\overline{\mathrm{P}}}^{\sigma} \\
y_{p}^{w}=0 & \forall(w, p) \in \Phi_{\overline{\mathrm{P}}}^{\sigma}
\end{array}
$$

where $\boldsymbol{\varepsilon} \geq 0$.

We use the solution of WC-BRUE as an example and introduce the ASA as follows.

Step 1: Let $\varpi=1$, and solve for the PRUE state of the transportation network with $\mathbf{u}=\mathbf{0}$ by applying the technique proposed by Xin et al. (2021). To avoid the enumeration of paths, one can learn from the CGA proposed in Section 5.3. To obtain good strongly stationary solutions, one may also apply this step with multiple initial solutions (Lou et al. 2010). Based on the model solution, set $\Phi_{\mathrm{P}}^{1}$ and $\Phi_{\overline{\mathrm{P}}}^{1}$, and go to

\section{Step 2.}

Step 2: Let vector $\left(\mathbf{v}^{\varpi}, \mathbf{f}^{\varpi}, \mathbf{y}^{\sigma}, \boldsymbol{\varepsilon}^{\sigma}, \lambda^{\sigma}\right)^{\mathrm{T}}$ solve the R-BC-BRUE model, and go to

\section{Step 3.}

Step 3: Set $\Pi_{\mathrm{P}}^{\sigma}:=\left\{(w, p) \in \Phi_{\mathrm{P}}^{\sigma} \cap \Phi_{\overline{\mathrm{P}}}^{\varpi}: v_{\varpi}^{(w, p)}>0\right\}$, where $v_{\bar{\sigma}}^{(w, p)}$ represents the multipliers associated with Constraint (21). If $\Pi_{\mathrm{P}}^{\sigma}=\varnothing$, then stop. At this point, the vector $\left(\mathbf{v}^{\sigma}, \mathbf{f}^{\sigma}, \mathbf{y}^{\sigma}, \boldsymbol{\varepsilon}^{\sigma}, \lambda^{\sigma}\right)^{\mathrm{T}}$ is the local optimal solution to the R-BC-BRUE problem; otherwise, go to Step 4. 
Step 4: Set $\Phi_{\mathrm{P}}^{\sigma+1}=\Phi_{\mathrm{P}}^{\sigma}-\Pi_{\mathrm{P}}^{\sigma}$ and $\Phi_{\overline{\mathrm{P}}}^{\sigma+1}=\left\{(w, p): y_{p}^{w}=0, \forall p \in P_{w}, w \in W\right\}$, and go to Step 5.

Step 5: Set $\varpi=\varpi+1$, and go to Step 2 .

\subsection{Column generation to avoid path enumeration}

According to our definition, not all paths are usable for an O-D pair in the BEV transportation network. This requires us to calculate the usable paths before solving for the BRUE state of the BEV transportation network to avoid enumerating the paths for each O-D pair. In this context, we apply the CGA proposed by Cheng et al. (2020). Before designing the algorithm, we need to develop a model and introduce the relevant notations for each O-D pair $w$. Let $\tilde{v}_{a}$ denote the traffic flow on link $a$. Since $v$ is a parameter in the model, a wavy line is added above it to distinguish the decision variable $v_{a}$. Similarly, $\tilde{u}_{(a, k)}$ is also a model parameter. $x_{a}^{w}$ is a binary variable; when the new path solved by the CGA for O-D pair $w$ contains link $a$, its value equals 1 ; otherwise, it equals $0 . \omega_{i}$ and $\pi_{i}$ denote the charging operation time (i.e., the time required by the driver to prepare before the BEV starts charging) and charging time at node $i$, respectively. $r_{i}^{w}$ is a binary variable indicating whether the BEV charges at node $i . F_{i}^{w}$ represents the amount of charge per unit time at node $i . \Delta$ is a node-link incidence matrix. $D_{w}$ is a vector of length $|N|$, where notation $|\cdot|$ denotes the cardinality of a set. Only two nonzero components are included in $D_{w}$ : the value of one component is 1 , and the that of the other is -1 . These components are associated with the origin (denoted as $o(w)$ ) and destination (denoted as $d(w))$ of O-D pair $w$, respectively. In terms of battery-related information for BEVs, we set $B_{0}$ and $B_{i}^{w}$ to 
denote the initial SOC of the BEV and the SOC at node $i \in N_{\mathrm{C}}$, respectively. $B_{\max }$ represents the maximum storage capacity of the battery. $d_{a}$ and $\vartheta$ denote the length of link $a$ and the energy consumption per unit distance, respectively. $\varphi_{a}^{w}$ is a variable and equals 1 only when $x_{a}^{w}=1 . R A_{w}$ indicates the lower limit of the SOC allowed by the driver, which is associated with range anxiety. $C_{i}$ represents the amount of charge that node $i \in N$ can provide. For nodes with charging stations, $C_{i}$ is a sufficiently large number; for nodes without charging stations, $C_{i}$ equals 0 . Finally, $K$ and $M$ are two sufficiently large positive numbers.

CGA: For each O-D pair $w$ :

$$
\begin{aligned}
& \min _{(\mathcal{X}, \mathbf{r}, \mathbf{F})} \sum_{a \in A} \sum_{k=1,2} t_{a}\left(\tilde{v}_{a}, \tilde{u}_{(a, k)}\right) x_{a}^{w}+\sum_{i \in N_{\mathrm{C}}}\left(\omega_{i} r_{i}^{w}+\pi_{i} F_{i}^{w}\right) \\
& \text { s.t. } \quad \Delta \mathcal{X}_{w}=D_{w} \\
& B_{j}^{w}-B_{i}^{w}+d_{a} \vartheta-F_{j}^{w}=\varphi_{a}^{w} \quad \forall(i, j)=a \in A \\
& B_{i}^{w}-d_{a} \vartheta \geq-M\left(1-x_{a}^{w}\right)+R A_{w} \quad \forall(i, j)=a \in A \\
& -K\left(1-x_{a}^{w}\right) \leq \varphi_{a}^{w} \leq K\left(1-x_{a}^{w}\right) \quad \forall a \in A \\
& 0 \leq F_{i}^{w} \leq C_{i} \quad \forall i \in N \\
& 0 \leq B_{i}^{w} \leq B_{\max } \quad \forall i \in N \\
& B_{o(w)}^{w}=B_{0} \\
& x_{a}^{w} \in\{0,1\} \quad \forall a \in A \\
& r_{i}^{w} \geq \frac{F_{i}^{w}}{C_{\max }} \quad \forall i \in N \\
& C_{\max }=\max _{i \in N_{\mathrm{C}}}\left\{C_{i}\right\}
\end{aligned}
$$




$$
r_{i}^{w} \in\{0,1\}
$$

$$
\forall i \in N
$$

where $\mathcal{X}_{w}=\left(x_{a}^{w}\right) ; \mathbf{r}=\left(r_{i}^{w}\right) ;$ and $\mathbf{F}=\left(F_{i}^{w}\right)$

Based on the above model, we propose the following method to explore the usable paths for each O-D pair by applying the CGA.

Step 1: Initialization. For each $w \in W$, solve the CGA with $\tilde{\mathbf{v}}=\left(\tilde{v}_{a}\right)=\mathbf{0}$. Set $P_{w}=\left\{p_{w}\right\}$ associated with $\mathcal{X}$, and calculate parameter $c_{p}^{w}$ associated with $p_{w}$; then, go to Step 2.

Step 2: Solve for the BRUE state of the network based on set $P_{w}$, and obtain the optimal solution $\left(\tilde{\mathbf{v}}, \tilde{\lambda}^{w}\right)^{\mathrm{T}}$; then, go to Step 3 .

Step 3: For each O-D pair $w \in W$, solve the CGA again, and obtain a new usable path $p_{w}$. If the objective value of the CGA $\sum_{a \in A} \sum_{k=1,2} t_{a}\left(\tilde{v}_{a}, \tilde{u}_{(a, k)}\right) x_{a}^{w}+\sum_{i \in N_{\mathrm{C}}}\left(\omega_{i} r_{i}^{w}+\pi_{i} F_{i}^{w}\right) \geq \tilde{\lambda}^{w}+\bar{\varepsilon}^{w}$ can be obtained for all O-D pairs, stop; in this case, vector $\left(\tilde{\mathbf{v}}, \tilde{\lambda}^{w}\right)^{\mathrm{T}}$ represents the BRUE flow distribution in the BEV transportation network. Otherwise, go to Step 2.

Note that CGA is an ordinary mixed-integer linear programming model that can be easily solved with commercial solvers such as CPLEX (CPLEX 2008). In addition, since the number of usable paths in a BEV transportation network is finite, the abovementioned procedure can be terminated after a finite number of iterations (Cheng et al. 2020; He et al. 2014).

\subsection{Solution procedure for the robust optimization model}

In this section, a heuristic algorithm is designed to solve the robust optimization model, 
i.e., the BDNPM. Since the inner problem and outer problem of the original model affect each other, we first consider separating these problems. Therefore, we convert BNDPM-IN into P-BNDPM-IN by adding a penalty function. With this approach, we can obtain an ordinary semi-infinite optimization problem that can be solved by the cutting-plane method proposed by Lawphongpanich and Hearn (2004).

P-BDNPM-IN(u):

$$
\max _{(\mathbf{v}, \mathbf{f}, \mathbf{y}, \boldsymbol{\varepsilon}, \lambda)} \sum_{a \in A} \sum_{k=1,2} t_{a}\left(v_{a}, u_{(a, k)}\right) v_{a}+\sum_{w \in W} \sum_{p \in P_{w}} c_{p}^{w} f_{p}^{w}-M \sum_{w \in W} \sum_{p \in P_{w}}\left(\bar{c}_{p}^{w}-\sum_{a \in A(p)} t_{a}\left(v_{a}, u_{(a, k)}\right)-c_{p}^{w}\right)^{2}
$$

s.t. (1), (5), (7), (9), (12), (13), and (14)

Let $\hat{\psi}(\Theta, \mathbf{z}, \mathbf{u})$ denote the objective function (37) and $\Xi$ denote the feasible region of the above model. If the penalty is infinite, then $\hat{\psi}(\Theta, \mathbf{z}, \mathbf{u})$ is an upper bound of $\psi(\Theta)$. For a feasible government lane expansion scheme $\mathbf{u}$, we let $\bar{\Theta}$ denote the solution vector to BNDPM-IN. $\hat{\Theta}$ represents the solution vector to P-BDNPM-IN(u) for some $M>0$. Then, we can obtain the following relationship.

$$
\left\{\begin{array}{l}
\psi(\bar{\Theta})=\hat{\psi}(\bar{\Theta}, \mathbf{u}) \\
\hat{\psi}(\bar{\Theta}, \mathbf{u}) \leq \hat{\psi}(\hat{\Theta}, \mathbf{u})
\end{array}\right.
$$

When $\hat{\psi}(\bar{\Theta}, \mathbf{u})$ is minimized, the penalty term in the objective function equals zero, so the equation in Equation (38) holds. Moreover, since $\bar{\Theta}$ is a feasible solution to P-BDNPM-IN(u), the inequality holds. Based on the above notations, we can develop a penalized BDNPM as follows.

BDNPM-P:

$$
\min _{\mathbf{u}} \max _{\Theta \in \Xi} \hat{\psi}(\Theta, \mathbf{u})
$$

Subject to Constraints (16), (17), and (18). 
BDNPM-P is an ordinary semi-infinite optimization problem because the feasible region of the inner problem (i.e., $\Xi$ ) is not related to the decision variable vector $\mathbf{u}$. To apply the cutting-plane method, we introduce an auxiliary variable $\sigma$ and convert BDNPM-P into BDNPM -P-1.

BDNPM-P-1:

$\min _{(\mathbf{u}, \sigma)} \sigma$

Subject to $\sigma \geq \hat{\psi}(\Theta, \mathbf{u})$ for all $\Theta \in \Xi$ and Constraints (16), (17), and (18).

Then, we use $\Theta^{1}, \Theta^{2}, \ldots, \Theta^{n}$ to represent the elements of $\Xi$ and propose a relaxed version of BDNPM-P-1, namely, R-BDNPM-P-1.

\section{R-BDNPM-P-1:}

$$
\min _{(\mathbf{u}, \sigma)} \sigma
$$

Subject to $\sigma \geq \hat{\psi}\left(\Theta^{i}, \mathbf{u}\right)$ for all $i=1,2, \ldots, n$ and Constraints (16), (17), and (18).

It should be noted that in R-BDNPM-P-1, set $\bar{\Xi}:=\left\{\Theta^{1}, \Theta^{2}, \ldots, \Theta^{n}\right\}$ is introduced to approximate $\Xi$. Let $(\tilde{\mathbf{u}}, \tilde{\sigma})$ denote a global optimal solution to R-BDNPM-P-1. If $(\tilde{\mathbf{u}}, \tilde{\sigma})$ is feasible for BDNPM-P-1, then it is also an optimal solution to R-BDNPM-P-

1. The feasibility of $(\tilde{\mathbf{u}}, \tilde{\sigma})$ can be verified by comparing $\hat{\psi}(\tilde{\Theta}, \tilde{\mathbf{u}})$ and $\tilde{\sigma}$. Specifically, if a solution $\tilde{\Theta}$ to $\operatorname{P-BDNPM-IN}(\mathbf{u})$ satisfies $\hat{\psi}(\tilde{\Theta}, \tilde{\mathbf{u}}) \leq \tilde{\sigma}$, then $(\tilde{\mathbf{u}}, \tilde{\sigma})$ is a feasible (also optimal) solution to BDNPM-P-1. Otherwise, $(\tilde{\mathbf{u}}, \tilde{\sigma})$ is infeasible for BDNPM-P1. At this time, R-BDNPM-P-1 should be solved again to obtain an improved solution with $\bar{\Xi}\{\tilde{\Theta}\}$. Based on the above descriptions, we give the solution procedure for the BNDPM as follows. 
Step 1: Initialization. Set $\mathbf{u}=\mathbf{0}$, and solve the WC-BRUE model to obtain an initial solution: $\Theta^{1}$. Set $\theta=1$ and $\bar{\Xi}^{\theta}=\left\{\Theta^{1}\right\}$, and go to Step 2 .

Step 2: Let $\left(\mathbf{z}^{\theta}, \mathbf{u}^{\theta}, \sigma^{\theta}\right)^{\mathrm{T}}$, and solve R-BDNPM-P-1 with $\bar{\Xi}^{\theta}$; then, go to Step 3 .

Step 3: Solve P-BDNPM-IN $\left(\mathbf{u}^{\theta}\right)$, and let $\Theta^{(\theta+1)}$ represent the optimal solution.

\section{Go to Step 4.}

Step 4: If $\hat{\psi}\left(\Theta^{(\theta+1)}, \mathbf{u}^{\theta}\right) \leq \sigma^{\theta}$, stop; in this case, $\mathbf{u}^{\theta}$ is an optimal robust lane expansion scheme. Otherwise, go to Step 5.

Step 5: Set $\bar{\Xi}^{(\theta+1)}=\bar{\Xi}^{\theta} \cup\left\{\Theta^{(\theta+1)}\right\}$ for $\theta=\theta+1$, and go to Step 2.

\section{Numerical example}

We perform numerical experiments based on the classic Sioux Falls network (Leblanc 1975; Long et al. 2010), as shown in Figure 2. This network encompasses 28 nodes and 76 links. The power limit of each electric vehicle is set to $40 \mathrm{kWh}$, and the initial SOC of all BEVs is $25 \mathrm{kWh}$. The power consumption per kilometer is set to $0.29 \mathrm{kWh}$ according to the value given by He et al. (2014). For the performance function, we set $\alpha_{1}=0.15$ and $\alpha_{2}=3$. The free-flow time and capacity parameters of the link are shown in Table 1. For each O-D pair, the range anxiety limit for BEV drivers is set to $0.1 \mathrm{kWh}$. The parameters in the CGA model are set as $\omega_{i}=0.3$ for all charging stations,

$\pi_{i}= \begin{cases}0.7, & \text { Level 1, } \\ 10, & \text { Level } 2, \mathrm{~min} / \mathrm{kWh} \text { and } B_{0}=0.25 B_{\max }(\text { He et al. 2014). The bounded } \\ 40, & \text { Level 3. }\end{cases}$ rationality parameter for traveler $\bar{\varepsilon}^{w}$ is set to $\alpha$ times the path travel time in the PRUE state. We assume that there are 5 nodes in the transportation network with charging piles. Node 11 and Node 15 are equipped with Level 1 charging piles. Node 5 and Node 
16 are equipped with Level 2 charging piles. At Node 12, several Level 3 charging piles are installed.
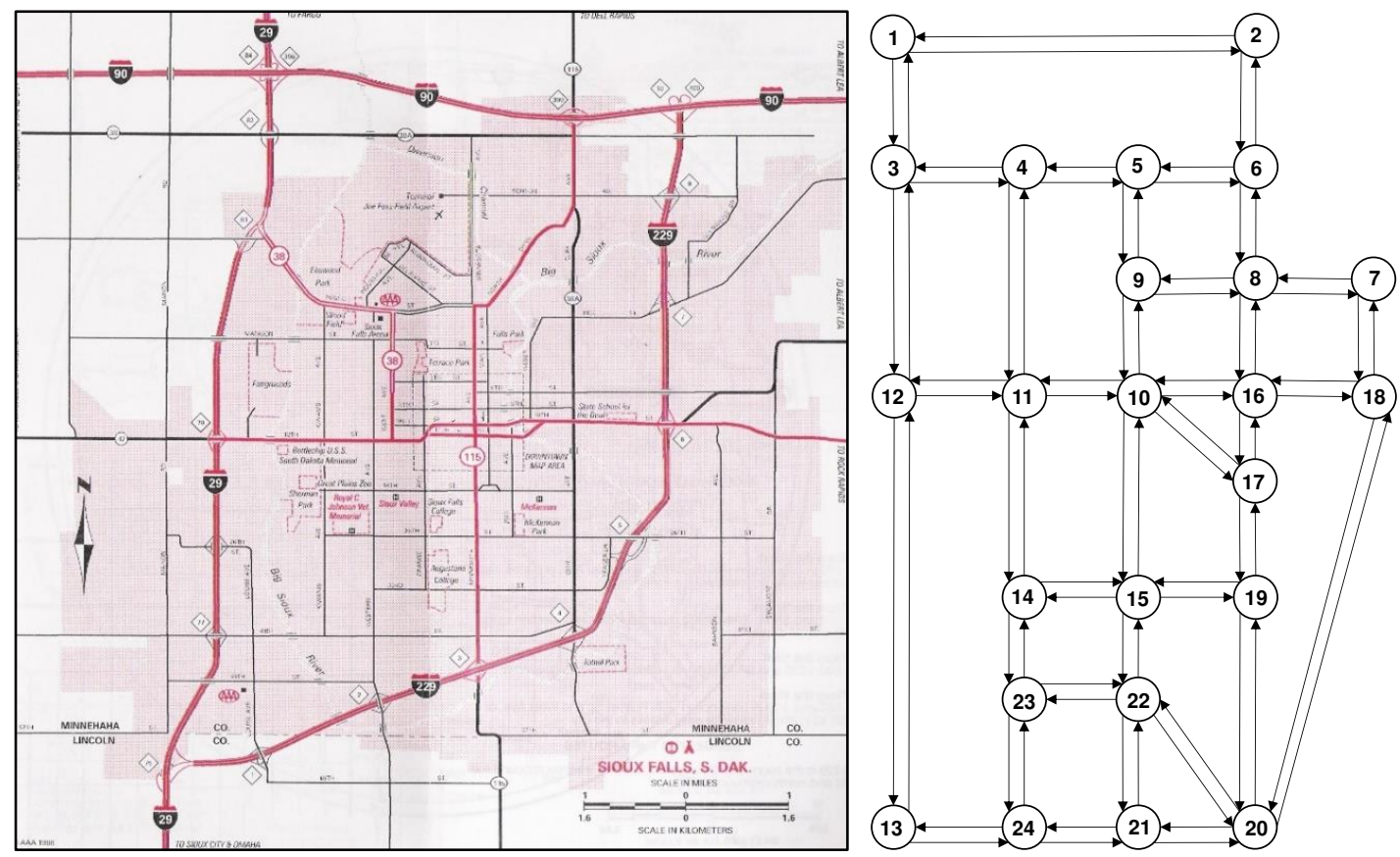

Fig. 2. Sioux Falls network (Source: He et al. (2020))

All the experimental procedures were run on a computer produced by Lenovo

with an Intel (R) Core (TM) i5-10210U CPU and 16 GB of available RAM. Among the various models constructed in this paper, the nonlinear programming models were solved with a commercial solver called CONOPT, the mixed-integer linear programming model was solved with CPLEX 12.4 (CPLEX 2008) and the MINLPs were solved with DICOPT (Kocis and Grossmann 1989). 
Table 1. Link capacities and free-flow travel times in the Sioux Falls network

\begin{tabular}{|c|c|c|c|c|c|}
\hline Link & $\begin{array}{c}t_{a}^{\text {free }} \\
\text { (minutes) }\end{array}$ & $\begin{array}{c}C A_{a} \\
\left(\times 10^{3} \text { vehicles } / \mathrm{h}\right)\end{array}$ & Link & $\begin{array}{c}t_{a}^{\text {free }} \\
\text { (minutes) }\end{array}$ & $\begin{array}{c}C A_{a} \\
\left(\times 10^{3} \text { vehicles } / \mathrm{h}\right)\end{array}$ \\
\hline 1 & 3.60 & 6.02 & 39 & 2.40 & 10.18 \\
\hline 2 & 2.40 & 9.01 & 40 & 2.40 & 9.75 \\
\hline 3 & 3.60 & 12.02 & 41 & 3.00 & 10.26 \\
\hline 4 & 3.00 & 15.92 & 42 & 2.40 & 9.85 \\
\hline 5 & 2.40 & 46.81 & 43 & 3.60 & 27.02 \\
\hline 6 & 2.40 & 34.22 & 44 & 3.00 & 10.26 \\
\hline 7 & 2.40 & 46.81 & 45 & 2.40 & 9.64 \\
\hline 8 & 2.40 & 25.82 & 46 & 2.40 & 20.63 \\
\hline 9 & 1.20 & 28.25 & 47 & 3.00 & 10.09 \\
\hline 10 & 3.60 & 9.04 & 48 & 3.00 & 10.27 \\
\hline 11 & 1.20 & 46.85 & 49 & 1.20 & 10.46 \\
\hline 12 & 2.40 & 13.86 & 50 & 1.80 & 39.36 \\
\hline 13 & 3.00 & 10.52 & 51 & 4.20 & 9.99 \\
\hline 14 & 3.00 & 9.92 & 52 & 1.20 & 10.46 \\
\hline 15 & 2.40 & 9.90 & 53 & 1.20 & 9.65 \\
\hline 16 & 1.20 & 21.62 & 54 & 1.20 & 46.81 \\
\hline 17 & 1.80 & 15.68 & 55 & 1.80 & 39.36 \\
\hline 18 & 1.20 & 46.81 & 56 & 2.40 & 8.11 \\
\hline 19 & 1.20 & 9.80 & 57 & 2.40 & 4.42 \\
\hline 20 & 1.80 & 15.68 & 58 & 1.20 & 9.65 \\
\hline 21 & 2.00 & 10.10 & 59 & 2.40 & 10.01 \\
\hline 22 & 3.00 & 10.09 & 60 & 2.40 & 8.11 \\
\hline 23 & 3.00 & 20.00 & 61 & 2.40 & 6.05 \\
\hline 24 & 2.00 & 10.10 & 62 & 3.60 & 10.12 \\
\hline 25 & 1.80 & 27.83 & 63 & 3.00 & 10.15 \\
\hline 26 & 1.80 & 27.83 & 64 & 3.60 & 10.12 \\
\hline 27 & 3.00 & 20.00 & 65 & 1.20 & 10.46 \\
\hline 28 & 3.60 & 27.02 & 66 & 1.80 & 9.77 \\
\hline 29 & 3.00 & 10.27 & 67 & 2.40 & 20.63 \\
\hline 30 & 4.20 & 9.99 & 68 & 3.00 & 10.15 \\
\hline 31 & 3.60 & 9.82 & 69 & 1.20 & 10.46 \\
\hline 32 & 3.00 & 20.00 & 70 & 2.40 & 10.00 \\
\hline 33 & 3.60 & 9.82 & 71 & 2.40 & 9.85 \\
\hline 34 & 2.40 & 9.75 & 72 & 2.40 & 10.00 \\
\hline 35 & 2.40 & 46.81 & 73 & 1.20 & 10.16 \\
\hline 36 & 3.60 & 9.82 & 74 & 2.40 & 11.38 \\
\hline 37 & 1.80 & 51.80 & 75 & 1.80 & 9.77 \\
\hline 38 & 1.80 & 51.80 & 76 & 1.20 & 10.16 \\
\hline
\end{tabular}

\subsection{Sensitivity analysis for the degree of bounded rationality}

In this section, we adjust the parameter $\alpha$ to observe the impact of different degrees of rational behavior on the vehicle distribution in the transportation network. We set the range of $\alpha$ from 0 to 0.20 and performed 20 experiments with 0.01 as the step size. The best/worst-case total travel times in different situations are shown in Figure 3 and Figure 4. As parameter $\alpha$ gradually increased, the system total travel time in the best 
case gradually decreased and tended to stabilize. Moreover, the system travel time for the worst case gradually increased. The above situation is in line with our expectations. As the parameter $\alpha$ increases, the choice behaviors of travelers in the system become increasingly irrational. Additionally, a traveler can make concessions to facilitate the travel of other individuals. Therefore, the system travel time in the best case will gradually tend to system optimal (SO) state. The opposite trend occurs in the worst case. With the increase in irrationality, the behaviors of travelers may hinder the travel of other individuals in extreme cases, thus increasing the total travel time. Therefore, the difference between the best and worst-case BRUE states increases. Specifically, the difference between the two increased from 100.26 minutes when $\alpha=0.01$ to 35,444 minutes when $\alpha=0.20$. These two values differ by a factor of more than 350 .

We further select the vehicle flow distribution for the best and worst cases with $\alpha=0.05$ and $\alpha=0.20$, and the results are shown in Table 2 and Table 3, respectively. Notably, as $\alpha$ increases, the flow distribution in the PRUE state will not change; however, the difference between the best and worst-case BRUE states becomes more obvious. For example, when $\alpha=0.05$, the flow difference for link $(1,2)$ in the best and worst cases is only 0.41 . However, when $\alpha$ increases to 0.20 , this flow difference increases to 6.67. The above phenomenon is consistent with the trend reflected in Figures 1 and 2. Another noteworthy phenomenon is that the flows along some links in Table 2 and Table 3 are 0 . Specifically, electric vehicles have limited mileage capacities, and the range anxiety of travelers may limit vehicle use. Moreover, some links may not be included in a usable path due to the particularities of the corresponding geographical locations. 


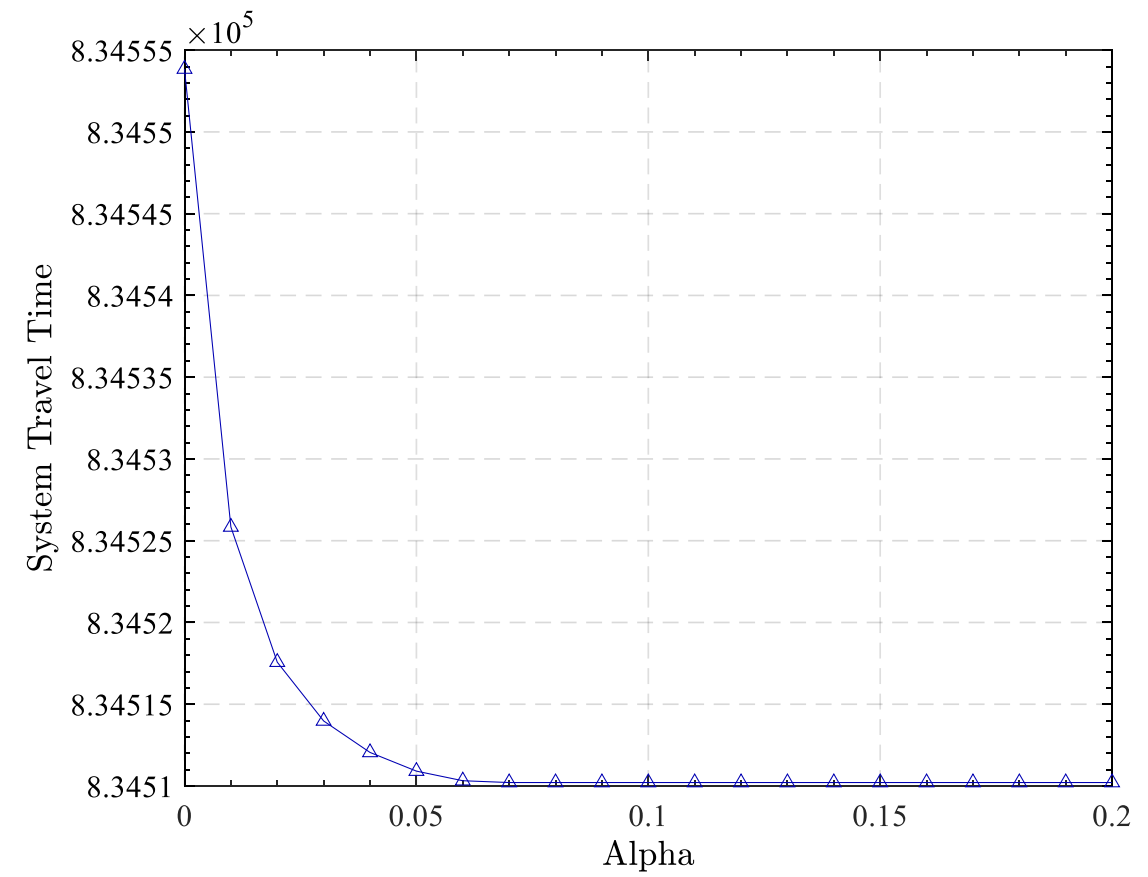

Fig. 3. Comparison of best-case total travel times in the network for different $\alpha$ values

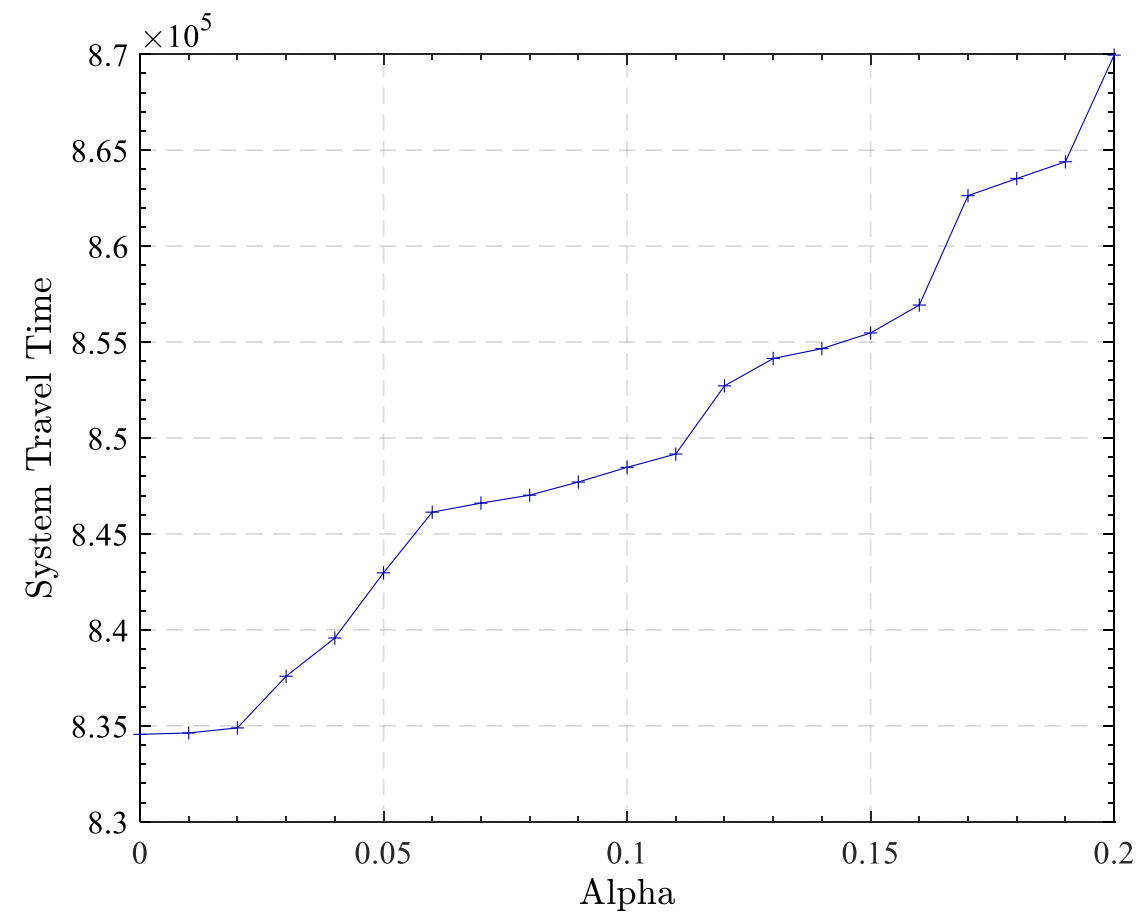

Fig. 4. Comparison of worst-case total travel times in the network for different $\alpha$ values 
Table 2. Flow distributions for the network $\left(\alpha=0.05, \times 10^{3}\right.$ vehicles $\left./ \mathrm{h}\right)$

\begin{tabular}{|c|c|c|c|c|c|c|c|c|c|c|c|}
\hline Link & PRUE & $\begin{array}{c}\text { WC- } \\
\text { BRUE }\end{array}$ & $\begin{array}{c}\text { BC- } \\
\text { BRUE }\end{array}$ & Link & PRUE & $\begin{array}{c}\text { WC- } \\
\text { BRUE }\end{array}$ & $\begin{array}{c}\text { BC- } \\
\text { BRUE }\end{array}$ & Link & PRUE & $\begin{array}{c}\text { WC- } \\
\text { BRUE }\end{array}$ & $\begin{array}{c}\text { BC- } \\
\text { BRUE }\end{array}$ \\
\hline$(1,2)$ & 224.67 & 225.73 & 225.32 & $(10,11)$ & 0.00 & 0.00 & 0.00 & $(17,19)$ & 66.88 & 77.35 & 67.11 \\
\hline$(1,3)$ & 475.33 & 474.27 & 474.68 & $(10,15)$ & 0.00 & 0.00 & 0.00 & $(18,7)$ & 167.01 & 144.08 & 169.15 \\
\hline$(2,1)$ & 51.40 & 53.30 & 51.46 & $(10,16)$ & 0.00 & 0.00 & 0.00 & $(18,16)$ & 148.60 & 146.70 & 148.54 \\
\hline$(2,6)$ & 274.67 & 275.73 & 275.32 & $(10,17)$ & 0.00 & 0.00 & 0.00 & $(18,2)$ & 51.40 & 53.30 & 51.46 \\
\hline$(3,1)$ & 0.00 & 0.00 & 0.00 & $(11,4)$ & 18.12 & 11.50 & 20.24 & $(19,15)$ & 66.88 & 77.35 & 67.11 \\
\hline$(3,4)$ & 173.83 & 222.83 & 171.93 & $(11,1)$ & 0.00 & 0.00 & 0.00 & $(19,17)$ & 108.06 & 120.23 & 107.83 \\
\hline$(3,12)$ & 526.54 & 512.76 & 526.09 & $(11,12)$ & 41.19 & 52.95 & 40.88 & $(19,2)$ & 0.00 & 0.00 & 0.00 \\
\hline$(4,3)$ & 51.22 & 93.05 & 51.41 & $(11,14)$ & 55.83 & 57.79 & 57.08 & $(20,18)$ & 0.00 & 0.00 & 0.00 \\
\hline$(4,5)$ & 325.67 & 367.85 & 324.79 & $(12,3)$ & 173.83 & 168.26 & 171.93 & $(20,19)$ & 0.00 & 0.00 & 0.00 \\
\hline$(4,11)$ & 72.85 & 71.81 & 74.18 & $(12,11)$ & 42.29 & 50.43 & 44.02 & $(20,21)$ & 0.00 & 0.00 & 0.00 \\
\hline$(5,4)$ & 157.79 & 198.38 & 158.22 & $(12,13)$ & 567.74 & 565.71 & 566.98 & $(20,22)$ & 0.00 & 0.00 & 0.00 \\
\hline$(5,6)$ & 139.18 & 130.76 & 138.01 & $(13,12)$ & 216.12 & 218.69 & 215.95 & $(21,2)$ & 0.00 & 0.00 & 0.00 \\
\hline$(5,9)$ & 86.50 & 87.09 & 86.78 & $(13,2)$ & 83.88 & 81.31 & 84.05 & $(21,22)$ & 44.15 & 38.66 & 43.82 \\
\hline$(6,2)$ & 33.73 & 38.09 & 32.62 & $(14$, & 0.00 & 0.00 & 0.00 & $(21,2$ & 37.56 & 41.82 & 37.22 \\
\hline$(6$, & 66.78 & 161.77 & 166.01 & (14, & 41.56 & 41.84 & 42.43 & (22, & 66.50 & 78.39 & 65.40 \\
\hline$(6,8)$ & 213.33 & 206.64 & 214.69 & $(14,23)$ & 36.11 & 36.92 & 37.70 & $(22,2)$ & 16.27 & 11.91 & 17.38 \\
\hline$(7,8)$ & 100.00 & 100.00 & 100.00 & $(15,1)$ & 0.00 & 0.00 & 0.00 & $(22,21)$ & 37.56 & 41.82 & 37.22 \\
\hline$(7,18)$ & 200.00 & 200.00 & 200.00 & $(15,14)$ & 4.45 & 18.06 & 4.40 & $(22,23)$ & 24.87 & 17.47 & 25.49 \\
\hline$(8,6)$ & 0.00 & 0.00 & 0.00 & $(15,19)$ & 108.06 & 120.23 & 107.83 & $(23,14)$ & 17.39 & 2.91 & 18.65 \\
\hline$(8,7)$ & 182.99 & 205.92 & 180.85 & $(15,22)$ & 62.42 & 59.29 & 62.70 & $(23,22)$ & 38.61 & 51.64 & 38.95 \\
\hline$(8,9)$ & 141.01 & 136.61 & 142.20 & $(16,8)$ & 0.00 & 32.53 & 0.00 & $(23,24)$ & 44.71 & 42.48 & 45.80 \\
\hline$(8,16)$ & 75.83 & 83.73 & 78.42 & $(16,1)$ & 71.30 & 74.40 & 70.07 & $(24,13)$ & 82.26 & 84.29 & 83.02 \\
\hline$(9,5)$ & 141.01 & 136.61 & 142.20 & $(16,17)$ & 94.17 & 99.65 & 95.58 & $(24,21)$ & 44.15 & 38.66 & 43.82 \\
\hline$(9,8)$ & 86.50 & 87.09 & 86.78 & $(16,18)$ & 167.01 & 144.08 & 169.15 & $(24,23)$ & 39.73 & 42.64 & 40.22 \\
\hline$(9,1)$ & 0.00 & 0.00 & 0.00 & $(17,1)$ & 27.29 & 22.30 & 28.47 & & & & \\
\hline$(10,9)$ & 0.00 & 0.00 & 0.00 & $(17,16)$ & 108.06 & 120.23 & 107.83 & & & & \\
\hline
\end{tabular}

Table 3. Flow distributions for the network $\left(\alpha=0.20, \times 10^{3}\right.$ vehicles $\left./ \mathrm{h}\right)$

\begin{tabular}{|c|c|c|c|c|c|c|c|c|c|c|c|}
\hline Link & PRUE & $\begin{array}{c}\text { WC- } \\
\text { BRUE }\end{array}$ & $\begin{array}{c}\text { BC- } \\
\text { BRUE }\end{array}$ & Link & PRUE & $\begin{array}{c}\text { WC- } \\
\text { BRUE }\end{array}$ & $\begin{array}{c}\text { BC- } \\
\text { BRUE }\end{array}$ & Link & PRUE & $\begin{array}{c}\text { WC- } \\
\text { BRUE }\end{array}$ & $\begin{array}{c}\text { BC- } \\
\text { BRUE }\end{array}$ \\
\hline$(1,2)$ & 224.67 & 231.99 & 225.32 & $(10,11)$ & 0.00 & 0.00 & 0.00 & $(17,19)$ & 66.88 & 87.94 & 67.11 \\
\hline$(1,3)$ & 475.33 & 468.01 & 474.68 & $(10,15)$ & 0.00 & 0.00 & 0.00 & $(18,7)$ & 167.01 & 127.30 & 169.15 \\
\hline$(2,1)$ & 51.40 & 56.77 & 51.46 & $(10,16)$ & 0.00 & 0.00 & 0.00 & $(18,16)$ & 148.60 & 143.23 & 148.54 \\
\hline$(2,6)$ & 274.67 & 281.99 & 275.32 & $(10,17)$ & 0.00 & 0.00 & 0.00 & $(18,2)$ & 51.40 & 56.77 & 51.46 \\
\hline$(3,1)$ & 0.00 & 0.00 & 0.00 & $(11,4)$ & 18.12 & 28.32 & 20.24 & $(19,15)$ & 66.88 & 87.94 & 67.11 \\
\hline$(3,4)$ & 173.83 & 220.42 & 171.93 & $(11,1)$ & 0.00 & 0.00 & 0.00 & $(19,17)$ & 108.06 & 127.30 & 107.83 \\
\hline$(3,12)$ & 526.54 & 491.97 & 526.09 & $(11,12)$ & 41.19 & 70.09 & 40.88 & $(19,2)$ & 0.00 & 0.00 & 0.00 \\
\hline$(4,3)$ & 51.22 & 100.00 & 51.42 & $(11,14)$ & 55.83 & 26.09 & 57.47 & $(20,18)$ & 0.00 & 0.00 & 0.00 \\
\hline$(4,5)$ & 325.67 & 372.70 & 324.40 & $(12,3)$ & 173.83 & 144.38 & 171.93 & $(20,19)$ & 0.00 & 0.00 & 0.00 \\
\hline$(4,11)$ & 72.85 & 70.09 & 74.57 & $(12,11)$ & 42.29 & 54.41 & 44.02 & $(20,21)$ & 0.00 & 0.00 & 0.00 \\
\hline$(5,4)$ & 157.79 & 194.05 & 158.22 & $(12,13)$ & 567.74 & 562.06 & 566.98 & $(20,22)$ & 0.00 & 0.00 & 0.00 \\
\hline$(5,6)$ & 139.18 & 114.49 & 137.62 & $(13,12)$ & 216.12 & 198.79 & 215.95 & $(21,2)$ & 0.00 & 0.00 & 0.00 \\
\hline$(5,9)$ & 86.50 & 108.21 & 86.79 & $(13,24)$ & 83.88 & 101.21 & 84.05 & $(21,22)$ & 44.15 & 8.09 & 43.82 \\
\hline$(6,2)$ & 33.73 & 50.00 & 32.23 & $(14,11)$ & 0.00 & 0.00 & 0.00 & $(21,24)$ & 37.56 & 28.43 & 37.22 \\
\hline$(6,5)$ & 166.78 & 146.43 & 166.01 & $(14,15)$ & 41.56 & 57.06 & 42.44 & $(22,15)$ & 66.50 & 70.24 & 65.40 \\
\hline$(6,8)$ & 213.33 & 300.04 & 214.69 & $(14,23)$ & 36.11 & 59.51 & 38.08 & $(22,2)$ & 16.27 & 0.00 & 17.77 \\
\hline$(7,8)$ & 100.00 & 100.00 & 100.00 & $(15,1)$ & 0.00 & 0.00 & 0.00 & $(22,21)$ & 37.56 & 28.43 & 37.22 \\
\hline$(7,18)$ & 200.00 & 200.00 & 200.00 & $(15,14)$ & 4.45 & 59.51 & 4.40 & $(22,23)$ & 24.87 & 0.00 & 25.49 \\
\hline$(8,6)$ & 0.00 & 100.00 & 0.00 & $(15,19)$ & 108.06 & 127.30 & 107.83 & $(23,14)$ & 17.39 & 30.97 & 18.65 \\
\hline$(8,7)$ & 182.99 & 222.70 & 180.85 & $(15,22)$ & 62.42 & 28.43 & 62.71 & $(23,22)$ & 38.61 & 62.15 & 39.34 \\
\hline$(8,9)$ & 141.01 & 147.62 & 142.20 & $(16,8)$ & 0.00 & 50.00 & 0.00 & $(23,24)$ & 44.71 & 59.51 & 45.80 \\
\hline$(8,16)$ & 75.83 & 87.94 & 78.42 & $(16,1)$ & 71.30 & 80.00 & 70.07 & $(24,13)$ & 82.26 & 87.94 & 83.02 \\
\hline$(9,5)$ & 141.01 & 147.62 & 142.20 & $(16,17)$ & 94.17 & 101.17 & 95.58 & $(24,21)$ & 44.15 & 8.09 & 43.82 \\
\hline$(9,8)$ & 86.50 & 108.21 & 86.79 & $(16,18)$ & 167.01 & 127.30 & 169.15 & $(24,23)$ & 39.73 & 93.12 & 40.22 \\
\hline$(9,1)$ & 0.00 & 0.00 & 0.00 & $(17,1)$ & 27.29 & 13.23 & 28.47 & & & & \\
\hline$(10,9)$ & 0.00 & 0.00 & 0.00 & $(17,16)$ & 108.06 & 127.30 & 107.83 & & & & \\
\hline
\end{tabular}




\subsection{Sensitivity analysis for the government investment scale}

In this section, we fix the parameter $\alpha$ and explore the changes in the lane expansion scheme for the BEV transportation network by adjusting the scale of government investment. We set $\alpha=0.10$, and the investment scale is increased from 0 to 100 in steps of 20. Therefore, six scenarios (C0 to $\mathrm{C} 5)$ are explored. The optimal lane expansion schemes and system travel times in different scenarios are shown in Table 4. The system travel times in different scenarios are also shown in Figure 5.

Notably, with increased government investment, the number of lane expansions significantly increases. The number of lane expansions increased from 4 in Scenario C1 to 24 in Scenario C5, an increase of $600 \%$. The system travel time was reduced by 13915.06 minutes; however, this decreasing trend was not significant. Specifically, the limited travel distance of BEVs and traveler anxiety limit the number of usable paths available in the BEV transportation network. Thus, the government could further reduce the travel time of vehicles in the system by simultaneously building charging stations and implementing lane expansion.

In addition, Figure 5 shows that the system travel time decreases most significantly when the investment amount increases from 20 to 40. Subsequently, the effect of investment is not as obvious as in the previous scenarios. This result suggests that government investment is characterized by diminishing marginal returns. In other words, with increasing government investment, the corresponding rate of decrease in system travel time will first increase and then decrease. Based on the current parameters, the government's optimal investment scale should be 40 . The above phenomenon indicates that for different investment goals, the government should scientifically set the investment scale to maximize social welfare. 
Table 4 The optimal lane expansion schemes in different scenarios

\begin{tabular}{|c|c|c|c|}
\hline Scenario & $\begin{array}{l}\text { Investment } \\
\text { scale }\end{array}$ & Optimal lane expansion scheme & $\begin{array}{l}\text { System travel } \\
\text { time for the } \\
\text { worst case } \\
\text { (minutes) }\end{array}$ \\
\hline $\mathrm{C} 0$ & 0 & None & 848468.903 \\
\hline $\mathrm{C} 1$ & 20 & $15-19$ (one lane), 23-22 (three lanes) & 846289.057 \\
\hline $\mathrm{C} 2$ & 40 & 2-6 (three lanes), 9-5(there lanes) & 840678.151 \\
\hline $\mathrm{C} 3$ & 60 & $\begin{array}{l}\text { 2-6 (three lanes), 4-11 (one lane), } 6-5 \text { (three lanes), 9-5 } \\
\text { (there lanes), 17-19 (one lane) }\end{array}$ & 838213.004 \\
\hline $\mathrm{C} 4$ & 80 & $\begin{array}{l}\text { 3-4 (three lanes), 3-12 (three lanes), } 4-5 \text { (three lanes), } \\
4-11 \text { (one lane), 6-8 (three lanes), 8-7 (two lanes), 8-9 } \\
\text { (one lane), 12-11 (one lane), 12-13 (three lanes) }\end{array}$ & 836600.272 \\
\hline $\mathrm{C} 5$ & 100 & $\begin{array}{l}\text { 3-4 (three lanes), 3-12 (three lanes), } 4-5 \text { (three lanes), } \\
4-11 \text { (one lane), 6-5 (one lane), 9-5 (one lane), 12-13 } \\
\text { (three lanes), 19-17 (one lane), 22-15 (two lanes), 23- } \\
22 \text { (three lanes), 24-23 (three lanes) }\end{array}$ & 834553.847 \\
\hline
\end{tabular}

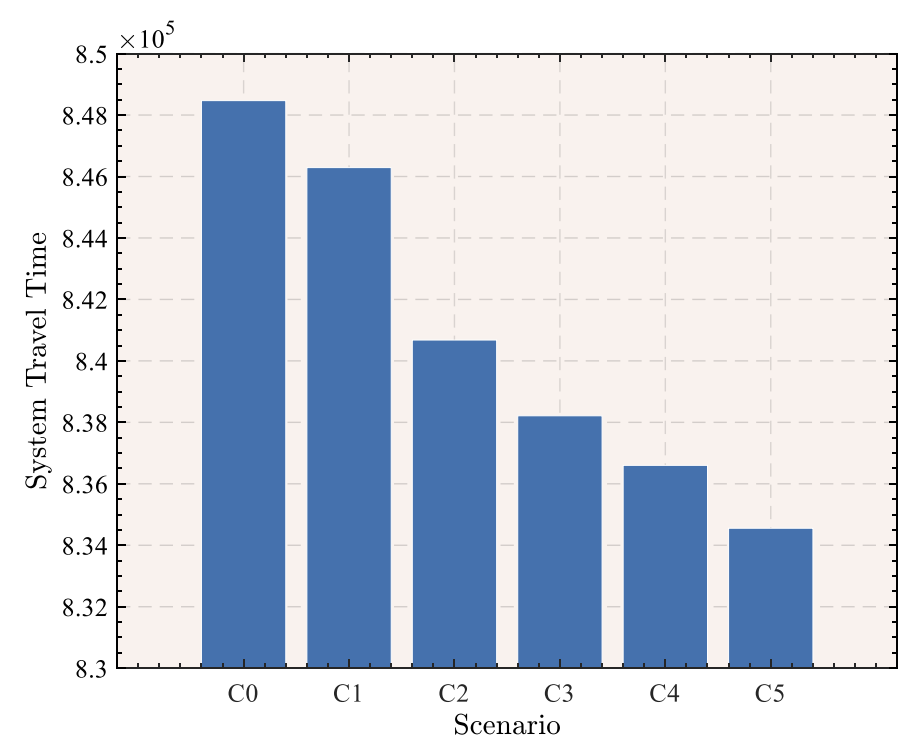

Fig. 5. System travel times under different scenarios

Figure 6 shows the government's lane expansion plans in different scenarios (Scenario C1 to Scenario C4) with red arrows. On the one hand, as the scale of government investment increases, the number of lanes included in expansion gradually increases. This finding is consistent with the results in the table. On the other hand, the links where the expanded lanes are located are almost all connected to nodes with charging stations (e.g., Node 5, Node 11, Node 12, and Node 16). This situation is in line with expectations. The links connected to nodes with charging stations are more likely to become components of usable paths. Moreover, as the scale of government investment increases, some links repeatedly appear in lane expansion schemes (e.g., 
links 2-6, 4-11, and 5-9). These links are the bottlenecks in the current transportation network. The government should focus on such bottleneck sections in the system and expand them first to effectively alleviate congestion in the entire network.
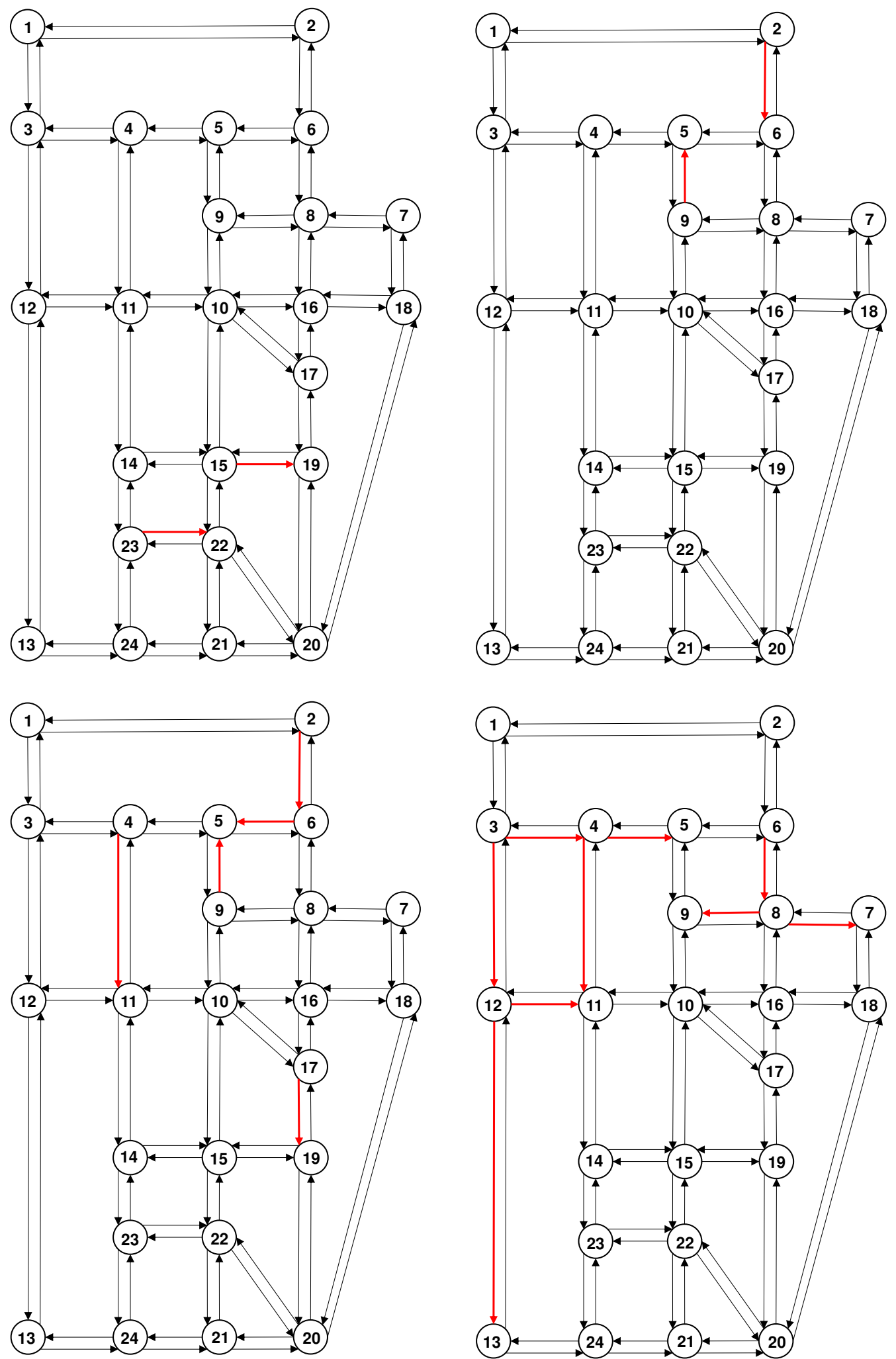

Fig. 6. Evolution of the lane expansion scheme: $\mathrm{C} 1 \rightarrow \mathrm{C} 2 \rightarrow \mathrm{C} 3 \rightarrow \mathrm{C} 4$ 


\section{Conclusions and recommendations}

In this paper, a BEV transportation network design problem is investigated. To address issues related to the mileage limitations of BEVs, traveler range anxiety, and bounded rationality, the problem is formulated as an MPCC. The model aims to minimize the total travel time in the transportation system and establish an optimal lane expansion scheme based on the available investment budget. A heuristic algorithm is proposed based on the framework of the ASA. Through column generation, we avoid the enumeration of paths in the transportation network.

Numerical tests are performed to assess the impacts of different traveler behaviors on travel times. Moreover, a sensitivity analysis of the investment scale is performed to explore the optimal level of government investment. The experimental results show that different levels of rationality among travelers influence the best- and worst-case scenarios for the flow distribution to various degrees. The greater the degree of irrationality is, the closer the best case is to the SO state of the BEV transportation network. Government investment is characterized by the law of diminishing marginal returns. Most of the links included in the investment scheme are connected to nodes with charging stations. Therefore, the experimental results verify that there are some bottleneck links in the BEV transportation network. Mitigating these bottlenecks is the key to improving the efficiency of the network. In addition, the solution approach can be efficiently used to support the proposed the network design scheme and can potentially be applied to even larger networks.

Overall, the model and algorithms we propose can effectively provide decisionmaking support for the government in infrastructure construction in BEV transportation networks. We extend the bounded rationality model proposed by Lou et al. (2010) for 
BEV transportation network design. Through the ongoing adjustment of the model, the robust joint optimization of congestion tolls and network design could be achieved.

Future work could focus on the collaborative optimization of different transportation network design schemes based on BEV characteristics. For example, the collaborative optimization of construction schemes for charging facilities (e.g., wireless charging roads and charging stations) considering bounded rationality is an interesting research direction. In addition, the electric energy consumption of BEVs varies for different traffic flows. Determining how to incorporate these variations into the proposed model framework is also a potential research direction.

\section{Acknowledgments}

The authors would like to express their gratitude for the support provided by the National Natural Science Foundation of China [grant numbers 72071025, 72072097, 72001120 and 72101129], the School-level Scientific Research Project of Beijing Wuzi University [grant number 2021XJKY10], the Shanghai Science and Technology Commission Project [grant number 18DZ1202700], the Social Science Planning Foundation of Liaoning [grant number 19BGL005], the Natural Science Foundation of Liaoning Province [grant number 2020-HYLH39] and the Special Foundation for Basic Scientific Research of the Central Colleges of China [grant number 3132021254]. Finally, the first author wants to thank Dazhou Yu for the support.

\section{Data availability statement}

All data, models, or codes that support the findings of this study are available from the corresponding author upon reasonable request.

\section{References}

Bogers, E. A., Viti, F., and Hoogendoorn, S. P. (2005). "Joint modeling of advanced travel information service, habit, and learning impacts on route choice by laboratory simulator experiments." Transportation Research Record, 1926(1), 189-197. 
Chen, R., Qian, X., Miao, L., and Ukkusuri, S. V. (2020). "Optimal charging facility location and capacity for electric vehicles considering route choice and charging time equilibrium." Computers \& Operations Research, 113, 104776.

Chen, Z., He, F., and Yin, Y. (2016). "Optimal deployment of charging lanes for electric vehicles in transportation networks." Transportation Research Part B: Methodological, 91, 344-365.

Cheng, K., Zou, Y., Xin, X., and Gong, S. (2020). "Optimal lane expansion model for a battery electric vehicle transportation network considering range anxiety and demand uncertainty." Journal of Cleaner Production, 276, 124198.

Chu, Z., Cheng, M., and Yu, N. N. (2021). "A smart city is a less polluted city." Technological Forecasting and Social Change, 172, 121037.

CPLEX, I. (2008). "11.0 Reference Manual." ILOG CPLEX Division, Gentilly.

Eikenbroek, O. A., Still, G. J., van Berkum, E. C., and Kern, W. (2018). "The boundedly rational user equilibrium: a parametric analysis with application to the network design problem." Transportation Research Part B: Methodological, 107, 1-17.

He, F., Yin, Y., and Lawphongpanich, S. (2014). "Network equilibrium models with battery electric vehicles." Transportation Research Part B: Methodological, 67, 306-319.

He, J., Yang, H., Tang, T.-Q., and Huang, H.-J. (2020). "Optimal deployment of wireless charging lanes considering their adverse effect on road capacity." Transportation Research Part C: Emerging Technologies, 111, 171-184.

Hu, L., Dong, J., and Lin, Z. (2019). "Modeling charging behavior of battery electric vehicle drivers: A cumulative prospect theory based approach." Transportation Research Part C: Emerging Technologies, 102, 474-489.

Ilin, R., and Rogova, G. L. "Decision-making involving low probability high consequence events under risk and uncertainty." Proc., 2017 IEEE Conference on Cognitive and Computational Aspects of Situation Management (CogSIMA), IEEE, 1-5.

KAI-INEMAN, D., and Tversky, A. (1979). "Prospect theory: An analysis of decision under risk." Econometrica, 47(2), 363-391.

Kocis, G. R., and Grossmann, I. E. (1989). "Computational experience with DICOPT solving MINLP problems in process systems engineering." Computers \& Chemical Engineering, 13(3), 307-315. 
Lawphongpanich, S., and Hearn, D. W. (2004). "An MPEC approach to second-best toll pricing." Mathematical programming, 101(1), 33-55.

Leblanc, L. J. (1975). "An algorithm for the discrete network design problem." Transportation Science, 9(3), 183-199.

Lin, B., Ghaddar, B., and Nathwani, J. (2021). "Electric vehicle routing with charging/discharging under time-variant electricity prices." Transportation Research Part C: Emerging Technologies, 130, 103285.

Liu, Z., and Song, Z. (2018). "Network user equilibrium of battery electric vehicles considering flow-dependent electricity consumption." Transportation Research Part C: Emerging Technologies, 95, 516-544.

Long, J., Gao, Z., Zhang, H., and Szeto, W. Y. (2010). "A turning restriction design problem in urban road networks." European Journal of Operational Research, 206(3), 569-578.

Lotan, T. (1997). "Effects of familiarity on route choice behavior in the presence of information." Transportation Research Part C: Emerging Technologies, 5(3-4), 225-243.

Lou, Y., Yin, Y., and Lawphongpanich, S. (2010). "Robust congestion pricing under boundedly rational user equilibrium." Transportation Research Part B: Methodological, 44(1), 15-28.

Mahmassani, H. S., and Chang, G.-L. (1986). "Experiments with departure time choice dynamics of urban commuters." Transportation Research Part B: Methodological, 20(4), 297-320.

Mahmassani, H. S., and Chang, G.-L. (1987). "On boundedly rational user equilibrium in transportation systems." Transportation science, 21(2), 89-99.

Miwa, T., Sato, H., and Morikawa, T. (2017). "Range and battery depletion concerns with electric vehicles." Journal of Advanced Transportation, 2017.

Montoya, A., Guéret, C., Mendoza, J. E., and Villegas, J. G. (2016). "A multi-space sampling heuristic for the green vehicle routing problem." Transportation Research Part C: Emerging Technologies, 70, 113-128.

Neubauer, J., and Wood, E. (2014). "The impact of range anxiety and home, workplace, and public charging infrastructure on simulated battery electric vehicle lifetime utility." Journal of power sources, 257, 12-20. 
Polak, E., and Royset, J. O. (2005). "On the use of augmented Lagrangians in the solution of generalized semi-infinite min-max problems." Computational Optimization and Applications, 31(2), 173-192.

Qiu, Y., Du, Y., Yu, S., and Jiang, S. (2020). "Location Design of Electrification Road in Transportation Networks for On-Way Charging." Journal of Advanced Transportation, 2020.

Royset, J. O., Polak, E., and Kiureghian, A. (2003). "Adaptive approximations and exact penalization for the solution of generalized semi-infinite min-max problems." SIAM Journal on Optimization, 14(1), 1-34.

Samuelson, W., and Zeckhauser, R. (1988). "Status quo bias in decision making." Journal of risk and uncertainty, 1(1), 7-59.

Schiffer, M., and Walther, G. (2017). "The electric location routing problem with time windows and partial recharging." European journal of operational research, 260(3), 995-1013.

Schwanen, T., and Ettema, D. (2009). "Coping with unreliable transportation when collecting children: examining parents' behavior with cumulative prospect theory." Transportation research part A: Policy and Practice, 43(5), 511-525.

Waraich, R. A., Galus, M. D., Dobler, C., Balmer, M., Andersson, G., and Axhausen, K. W. (2013). "Plug-in hybrid electric vehicles and smart grids: Investigations based on a microsimulation." Transportation Research Part C: Emerging Technologies, 28, 74-86.

Wardrop, J. G. (1952). "Correspondence. some theoretical aspects of road traffic research." Proceedings of the Institution of Civil Engineers, 1(5), 767-768.

World Meteorological (2021). "The Atlas of Mortality and Economic Losses from Weather, Climate and Water Extremes (1970-2019)."

Xin, X., Wang, X., Ma, L., Chen, K., and Ye, M. (2021). "Shipping network designinfrastructure investment joint optimization model: a case study of West Africa." Maritime Policy \& Management, 1-27.

Xu, M., Meng, Q., and Liu, K. (2017). "Network user equilibrium problems for the mixed battery electric vehicles and gasoline vehicles subject to battery swapping stations and road grade constraints." Transportation Research Part B: Methodological, 99, 138-166. 
Xu, M., Wu, T., and Tan, Z. (2021). "Electric vehicle fleet size for carsharing services considering on-demand charging strategy and battery degradation." Transportation Research Part C: Emerging Technologies, 127, 103146.

Xu, M., Yang, H., and Wang, S. (2020). "Mitigate the range anxiety: Siting battery charging stations for electric vehicle drivers." Transportation Research Part C: Emerging Technologies, 114, 164-188.

Yang, Y., Yao, E., Yang, Z., and Zhang, R. (2016). "Modeling the charging and route choice behavior of BEV drivers." Transportation Research Part C: Emerging Technologies, 65, 190-204.

Yuan, Q., Hao, W., Su, H., Bing, G., Gui, X., and Safikhani, A. (2018). "Investigation on range anxiety and safety buffer of battery electric vehicle drivers." Journal of Advanced Transportation, 2018.

Zhao, X., Shang, Y., and Song, M. (2020). "Industrial structure distortion and urban ecological efficiency from the perspective of green entrepreneurial ecosystems." Socio-Economic Planning Sciences, 72, 100757.

Zhou, L., Zhong, S., Ma, S., and Jia, N. (2014). "Prospect theory based estimation of drivers' risk attitudes in route choice behaviors." Accident Analysis \& Prevention, 73, 1-11. 